\title{
In-flight performance of the Ozone Monitoring Instrument
}

\author{
V. M. Erik Schenkeveld ${ }^{1}$, Glen Jaross ${ }^{2}$, Sergey Marchenko ${ }^{3}$, David Haffner ${ }^{3}$, Quintus L. Kleipool ${ }^{1}$, \\ Nico C. Rozemeijer ${ }^{4}$, J. Pepijn Veefkind ${ }^{1,5}$, and Pieternel F. Levelt ${ }^{1,5}$ \\ ${ }^{1}$ Royal Netherlands Meteorological Institute (KNMI), De Bilt, the Netherlands \\ ${ }^{2}$ NASA Goddard Space Flight Center, Greenbelt, Maryland, USA \\ ${ }^{3}$ Science Systems and Applications Inc., Lanham, Maryland, USA \\ ${ }^{4}$ TriOpSys BV, Utrecht, the Netherlands \\ ${ }^{5}$ Faculty of Civil Engineering and Geosciences, Delft University of Technology, Delft, the Netherlands
}

Correspondence to: Quintus L. Kleipool (quintus.kleipool@knmi.nl)

Received: 29 December 2016 - Discussion started: 11 January 2017

Revised: 29 March 2017 - Accepted: 7 April 2017 - Published: 1 June 2017

\begin{abstract}
The Dutch-Finnish Ozone Monitoring Instrument (OMI) is an imaging spectrograph flying on NASA's EOS Aura satellite since 15 July 2004 . OMI is primarily used to map trace-gas concentrations in the Earth's atmosphere, obtaining mid-resolution $(0.4-0.6 \mathrm{~nm})$ ultraviolet-visible (UVVIS; 264-504 nm) spectra at multiple (30-60) simultaneous fields of view. Assessed via various approaches that include monitoring of radiances from selected ocean, land ice and cloud areas, as well as measurements of line profiles in the solar spectra, the instrument shows low optical degradation and high wavelength stability over the mission lifetime. In the regions relatively free from the slowly unraveling "row anomaly" (RA) the OMI irradiances have degraded by $3-$ $8 \%$, while radiances have changed by $1-2 \%$. The long-term wavelength calibration of the instrument remains stable to $0.005-0.020 \mathrm{~nm}$.
\end{abstract}

\section{Introduction}

The Dutch-Finnish Ozone Monitoring Instrument (OMI) is an imaging spectrograph flying onboard the NASA's EOS Aura satellite since 15 July 2004. OMI is used to measure atmosphere trace gases $\left(\mathrm{O}_{3}, \mathrm{NO}_{2}, \mathrm{SO}_{2}, \mathrm{HCHO}\right.$, etc.), aerosol characteristics and various other quantities (e.g., surface UV radiation). The instrument has previously been described in Levelt et al. (2006), its calibration in Dobber et al. (2006) and the Level 1B data processor in Oord et al. (2006). OMI continues to extend the 25-year record of ozone measurements that started with the SBUV (Cebula et al., 1988) and TOMS
(McPeters et al., 1998) instruments of the National Aeronautics and Space Administration (NASA). This record has been continued by measurements from the SCIAMACHY (Bovensmann et al., 1999), GOME (Burrows et al., 1999) and GOMOS (Kyrölä et al., 2004) instruments of the European Space Agency (ESA) and EUMETSAT. Currently the OMPS instrument (Seftor et al., 2013) of NASA is also performing ozone measurements. After the end-of-life of OMI, the ozone record will be continued by the future instruments Tropomi (Veefkind et al., 2012), Sentinel 4 (Bazalgette Courrèges-Lacoste et al., 2008) and Sentinel 5 (Sierk et al., 2012) from ESA, GEMS from South Korea (Kim et al., 2015) and TEMPO from NASA (Chance et al., 2013). Tropomi and Sentinel 5 will be in a polar, Sun-synchronous orbit comparable to the OMI orbit. Sentinel 4, TEMPO and GEMS will be in a geostationary orbit, enabling them to monitor regions of the Earth with high temporal (e.g., diurnal) resolution. It is estimated that the product results will be available every hour during daytime, whereas OMI, Tropomi and Sentinel 5 only have a product delivery once per day. The older instruments needed even more time to get global coverage. At the time of writing of this article OMI continued to provide high-quality science data. This article focuses on the long-term OMI performance, primarily addressing results of in-flight calibration and changes in the instrument during the mission.

The instrument measures Earth radiances and solar irradiances which are stored in Level 1 products (Sect. 1.1) and used by various Level 2 retrieval algorithms to estimate ozone, trace gases and aerosol properties and UV irradiance. A brief sketch of the optical design (Sect. 2.1) is followed 


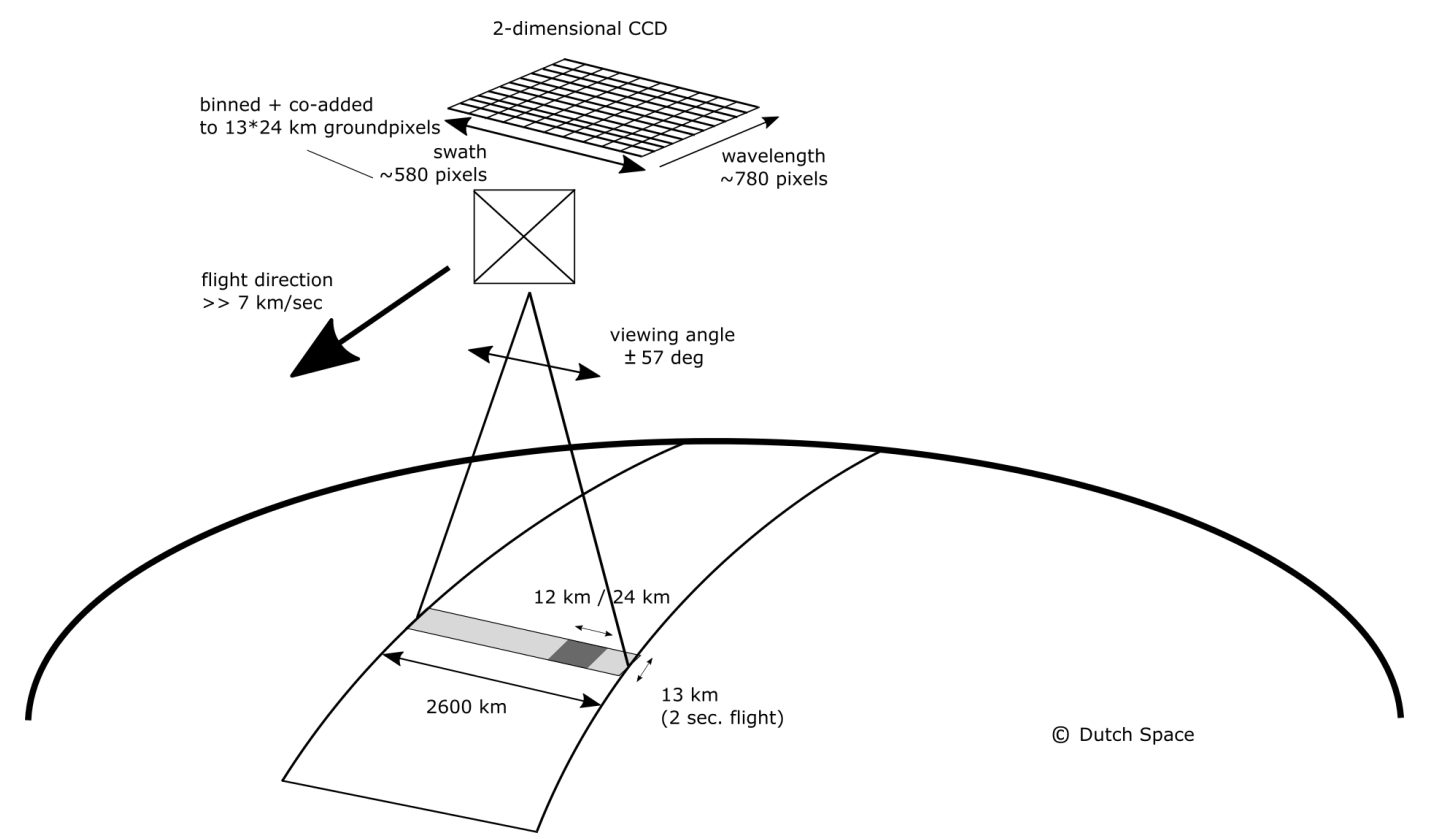

Figure 1. An impression of OMI flying over the Earth. The spectrum of a ground pixel is projected on the wavelength dimension of the charge-coupled device (CCD; the columns). The cross-track ground pixels are projected on the swath dimension of the CCD (the rows). The forward speed of $7 \mathrm{~km} \mathrm{~s}^{-1}$ and an exposure time of $2 \mathrm{~s}$ lead to a ground pixel size of $13 \mathrm{~km}$ in the flight direction. The viewing angle of $114^{\circ}$ leads to a swath width on the ground of $2600 \mathrm{~km}$.

Table 1. Optical properties for the three channels UV1, UV2 and VIS.

\begin{tabular}{lrrrr}
\hline Channel & Wavelength range & Spectral resolution & Spectral sampling & Ground pixel size \\
\hline UV1 & $264-311 \mathrm{~nm}$ & $0.63 \mathrm{~nm}=1.9 \mathrm{px}$ & $0.33 \mathrm{~nm} \mathrm{px}^{-1}$ & $13 \times 48 \mathrm{~km}$ \\
UV2 & $307-383 \mathrm{~nm}$ & $0.42 \mathrm{~nm}=3.0 \mathrm{px}$ & $0.14 \mathrm{~nm} \mathrm{px}^{-1}$ & $13 \times 24 \mathrm{~km}$ \\
VIS & $349-504 \mathrm{~nm}$ & $0.63 \mathrm{~nm}=3.0 \mathrm{px}$ & $0.21 \mathrm{~nm} \mathrm{px}^{-1}$ & $13 \times 24 \mathrm{~km}$ \\
\hline
\end{tabular}

Table 2. Standard OMI Level 1 data products.

\begin{tabular}{ll}
\hline Product & Description \\
\hline OML1BRUG & Global UV radiance product \\
OML1BRVG & Global VIS radiance product \\
OML1BRUZ & Spatial zoom-in UV radiance product \\
OML1BRVZ & Spatial zoom-in VIS radiance product \\
OML1BIRR & Irradiance product \\
OML1BCAL & Calibration product \\
\hline
\end{tabular}

by description of the charge-coupled device (CCD) detectors (Sect. 2.2). Section 3 provides details of the regular calibration routines, including wavelength calibration. A more detailed description of the wavelength registration algorithms is provided in the Appendix. Section 4 describes the basic instrument performance, such as white light and LED calibration sources (Sect. 4.1) as well as long-term changes in the CCDs (Sect. 4.2): gains, electronic offsets, linearity, dark currents, random telegraph noise, bad pixels, signal-to- noise ratio, and pixel response non-uniformity (PRNU). Section 4.3 discusses the approach to the evaluation of and corrections for stray light. The changes in the instrument temperatures and voltages are described in Sect. 4.4 and 4.5. Section 5 concerns the so-called "row anomaly" (RA), its evolution since June 2007, and its impact on the measured radiances. Section 6 provides some basic details of the current (Collection 3) approach to radiometric calibration, and in particular it describes the long-term changes in instrument throughput as observed in solar irradiance measurements performed using a set of diffusers and complemented by measurements of Earth radiances from sites with useful geophysical conditions (e.g., clouds, open-water and ice fields). Section 6 also describes the results of a comprehensive assessment of the instrument transfer function. The conclusions of our study are provided in Sect. 7.

\subsection{Basic goals and requirements for Level 1 products}

The OMI spectrograph acquires mid-resolution $(0.4-0.6 \mathrm{~nm})$ spectra in the $264-504 \mathrm{~nm}$ wavelength range. This spectral 


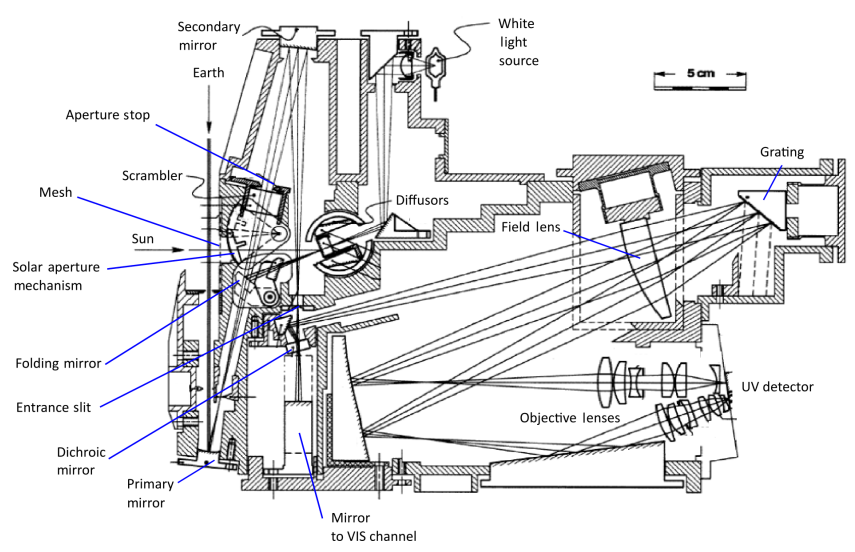

Figure 2. Optical design of the UV channel. The telescope (primary and secondary mirror) is used for both channels. The visible light that passes the dichroic mirror is coupled into the VIS channel. The folding mirror is depicted in two positions (Earth view and Sun/calibration view).

region is measured by three instrument channels, UV1, UV2 and VIS (visible; Table 1). For the UV2 and VIS channel the spectral sampling is 3 pixels for the full width at half maximum (FWHM). For the UV1 channel this is 1.9 pixel for the FWHM, which implies that the UV1 channel is undersampled. This is not a problem for operational use of OMI, because the UV1 channel is mainly used for ozone profile retrieval, which uses absolute radiances, and does not rely on spectral fitting. The wide, $115^{\circ}$ swath angle permits a ground track with a swath width of $2600 \mathrm{~km}$. This broad swath is simultaneously imaged in 60 cross-track field-of-view (FOV) channels (detector rows) for the UV2 and VIS channels and 30 for the UV1 channel. OMI follows a polar, Sun-synchronous orbit with an average altitude of $705 \mathrm{~km}$, orbital period of $98 \mathrm{~min} 53 \mathrm{~s}$ and an ascending node local time of 13:42 (note that the equator-crossing time was slightly adjusted during the mission). OMI orbits the Earth 14 times per day, providing daily global coverage in nominal operation. The short exposure times (typically, around $2 \mathrm{~s}$ ) result in a spatial resolution of $13 \mathrm{~km}$ in the flight direction.

There are six standard OMI Level 1 data products (Table 2). The products used to generate Level 2 products are generally the global UV and VIS radiance products and accompanying irradiance data. The spatial zoom products are produced 1 day per month. These products zoom in on a smaller swath on ground with a higher spatial resolution. The irradiances are measured once per day. The calibration product provides, for each orbit, the dark-current, background, WLS (white light source) and LED measurements and, whenever applicable (i.e., only at the times of solar calibration), the solar irradiance measurements in different formats, which supplements the standard irradiance output.

The quality of information in Level 1 data products is a somewhat relative concern from the standpoint of different

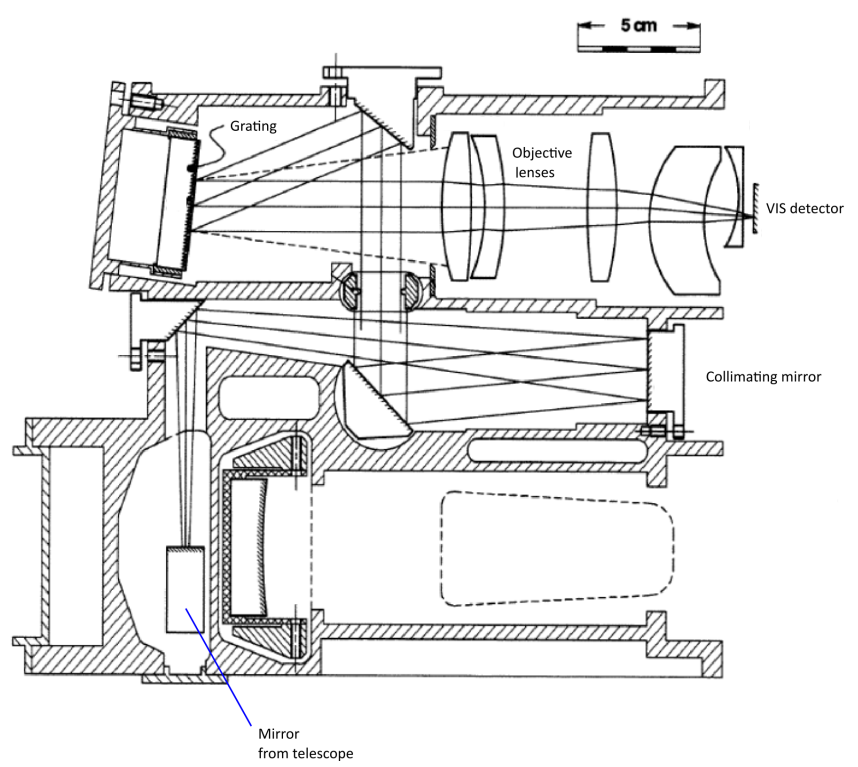

Figure 3. Optical design of the VIS channel. The light coming from the telescope (not shown) enters the VIS channel via the mirror from the telescope.

retrieval applications, since there is considerable variety in the sensitivity of different retrievals to errors and instrument degradation in the Level 1 data. A full review of these sensitivities is beyond the present scope of this paper, but, where appropriate, we summarize them for additional context.

\section{Instrument design}

In this section a description of the optical (Figs. 2 and 3) and detector (Fig. 4) design of the Ozone Monitoring Instrument is presented. The Ozone Monitoring Instrument (Levelt et al., 2006; Dobber et al., 2006) is a nadir viewing imaging spectrograph where the UV and visible range of the Earth spectrum is imaged onto two CCD detectors. One dimension of each CCD detector is used for the wavelength measurement, and the other dimension is used for spatial measurement of the cross-track field of view perpendicular to the flight direction. An impression of OMI flying over the Earth surface illustrates the flight and measurement configuration in Fig. 1.

\subsection{Optical design}

The Earth radiances and solar irradiances are acquired through mostly identical optical pathways.

\subsubsection{Radiance channel}

The Earth radiance is imaged via the telescope (primary and secondary mirror) onto the entrance slit. A polarization scrambler is placed in the vicinity of aperture stop and before the secondary mirror of the telescope. The secondary 

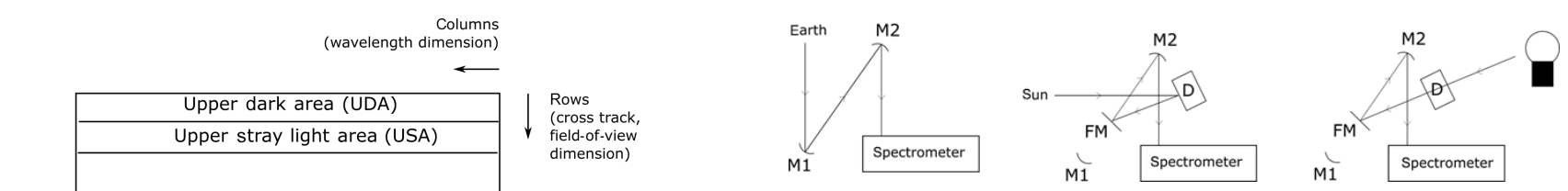

Figure 5. Schematic optical path. The left panel shows the configuration for Earth radiance measurements. M1 and M2 correspond to the primary and secondary mirrors of the telescope. The middle panel shows the configuration for Sun irradiance measurements, with the folding mirror FM placed between M1 and M2. Sunlight enters the instrument via diffuser D in reflection mode and the FM and M2. The right panel shows the configuration for internal calibration measurements. The light from the white light source passes diffuser $\mathrm{D}$ in transmission mode and enters the instrument via FM and $\mathrm{M} 2$.

is opened. This is done once per day just before the spacecraft enters into eclipse, at the northern part of the Earth. The sunlight is then reflected by one of the reflection diffusers: quartz volume diffuser (QVD), regular Aluminum or backup Aluminum. These diffusers are mounted on a diffuser carousel. The QVD diffuser is used in daily irradiance measurements. To monitor degradation of the QVD diffuser, the regular $\mathrm{Al}$ diffuser is used once per week and the backup $\mathrm{Al}$ diffuser once per month. After being reflected by the diffuser, the light can be reflected by the folding mirror (FM), once the mirror is moved to the Sun-observing position, thus blocking the Earth light. After reflection by the folding mirror the optical path is identical for radiance and irradiance. Thus, the difference between the radiance and irradiance optical pathways is the primary mirror for the radiance channel and the reflection diffuser and folding mirror for the irradiance channel. UV2 channels are split, because the requirements for these channels are different. The UV1 channel is designed to detect the shortest wavelengths. This channel is primarily used for the detection of ozone profiles. The UV signal decreases rapidly for shorter wavelengths, resulting in a low signal-tonoise ratio (SNR). To increase the SNR a design choice was made to increase the detector pixel size, at the expense of spatial resolution. The result is that only 30 spatial channels are available for UV1, versus 60 for UV2 and VIS. Also the choice of coatings on the optical elements is optimized for each spectral channel. Two sets of objective lenses project the spectrum onto the UV CCD detector. In the VIS channel a set of mirrors project the VIS signal onto the grating. The objective lenses project the image of the spectrum onto the VIS CCD detector.

\subsubsection{Irradiance channel}

In the irradiance channel the first component the sunlight passes is the optical mesh with $10 \%$ transmission. The sunlight can enter the instrument if the solar aperture mechanism

\subsubsection{Calibration channel}

OMI is equipped with two calibration light sources: a quartz tungsten halogen (QTH) white light source and green LEDs. The WLS is imaged via a lens and two mirrors onto a transmission diffuser, which is mounted on the diffuser carousel. When the WLS is used for measurements, the folding mirror is placed in the calibration position. This position will block the Earth radiance. The WLS is used to measure changes in the CCD performance, in particular the pixel-to-pixel response non-uniformity. The WLS can also be used to monitor radiometric throughput. Both the UV and VIS channel are equipped with two green LEDs. These LEDs are placed just before the CCD detector. In the VIS channel the LED light passes directly through the channel objective; in the UV channel the illumination is indirect. The LEDs can be used to monitor the CCD pixel behavior and linearity of the detector and electronics. 


\subsection{Detectors and electronics unit}

The OMI instrument is equipped with two CCD detectors and one electronics unit (ELU). The CCD detectors in the UV and VIS channel are back-illuminated UV-enhanced silicon-based CCDs. These detectors (see more details in Dobber et al., 2006) have 780 (spectral; hereafter designated as column) $\times 576$ (spatial, or row) pixels. The operational temperature of the CCDs is $265 \mathrm{~K}$, stabilized with a precision of $\pm 10 \mathrm{mK}$. The ELU controls and reads out both CCD detectors. The detectors have basic readout electronics and a programmable gain. The relative gain values can be G1, G4, G10 and G40, respectively, and can be programmed for certain column (i.e., spectral) ranges, thus providing extended dynamic range, critically important in the UV spectral region affected by strong ozone absorption. Three different gain switch columns can be used per CCD detector. Figure 4 shows the UV CCD detector layout. The CCD detector is divided in different parts. The useful (ir)radiance signal is detected by the UV1 and UV2 areas in the image area. Above and below are stray-light areas, designated for dynamic estimates of stray-light levels. At the extreme ends (rows) of the image area are dark-current areas. During readout the entire image area is transferred to the storage area. From there the image is read out via the readout register. During the readout a new image is acquired in the image area. The VIS channel carries similar designated areas.

\section{Calibration concept and implementation}

Calibration measurements for OMI are performed every day. These comprise solar, background and dark-current measurements as well as the data from dedicated onboard stimuli. The optical paths for radiance, irradiance and calibration measurements are almost identical, except for a few elements (Fig. 5).

For radiance measurements both the primary and secondary mirror, M1 and M2, of the telescope are used. For calibration measurements the folding mirror, FM, is put in the light path between M1 and M2, effectively blocking the Earth shine. Solar light enters the instrument via the diffuser $\mathrm{D}$ in reflection mode. Calibration measurements with the internal white light source use almost the same configuration as the solar calibration. The only difference is that the internal calibration light passes through the diffuser in transmission mode. Radiance and calibration pathways comprise the same optical elements, except mirror M1 for radiance and the diffuser D and the folding mirror FM for calibration measurements. Thus, in general these calibration pathways are suitable for calibration and degradation monitoring of all optical elements except mirror M1.

\subsection{Calibration data analysis}

Below we discuss the results of analysis of the L1B and telemetric data performed in three different ways. The Trend Monitoring and Calibration Facility (TMCF; hosted by KNMI, the Netherlands, see TMCF, 2006) performs basic analysis of daily L1B and telemetry data. In the second approach we evaluate the widths, depths and wavelength positions of well-defined absorption features (usually, blends of spectral lines) in the solar and earthshine spectra. Lastly, we analyze long-term trends in the OMI radiances observed over various geographical areas as well as relatively small changes in the daily irradiance measurements. In particular, we pay attention to a sub-set of data acquired over the ice fields of Greenland and Antarctica, i.e., the regions with relatively stable, spatially homogeneous and predictable reflectances.

\subsection{Wavelength registration approach}

There are two wavelength registration approaches used in the OMI radiances and irradiances. Hence, the L1B OMI products provide two slightly different wavelength grids. Here we briefly summarize the algorithms, providing more detailed discussion in Appendix A.

During the preflight testing and characterization the wavelength calibration was performed using a PtCrNeAr spectral line source (Dobber et al., 2006). Narrow wavelength windows were centered on prominent spectral lines with accurately known wavelengths: five lines in the UV1 channel, nine UV2 and nine VIS lines. In each window the observed line profile was fitted (in the CCD pixel space) with a Gaussian function in the UV1 channel and a sum of a Gaussian and a flat top function in the UV2 and VIS channels. The fits provided three sets (one per channel) of line-peak positions which were fitted with a fourth-order polynomial and translated into wavelength grids, thus providing the wavelength value for a given CCD pixel (column number).

In-orbit wavelength assignment for radiances and irradiances is done using two methods. In the first approach the wavelength assignment is based on pre-launch and early inorbit wavelength calibration parameters, i.e., the polynomial coefficients modified as a function of optical bench (OPB) temperature. For the UV2 and VIS channel this function is corrected for wavelength shifts that result from inhomogeneous slit illumination (see more details in the Appendix A). The result is a wavelength map:

$\lambda(i, j)=\sum_{n=0}^{N} c_{2, n}(j)\left(i-i i_{\mathrm{ref}}\right)^{n}$,

where $i$ is column number, $j$ is row number, $n$ is the number of polynomial coefficients (typically 4 ) and $i i_{\text {ref }}$ is a reference column for the spectral calibration polynomial coefficients. The wavelength coefficients $c(j)$ and the reference 


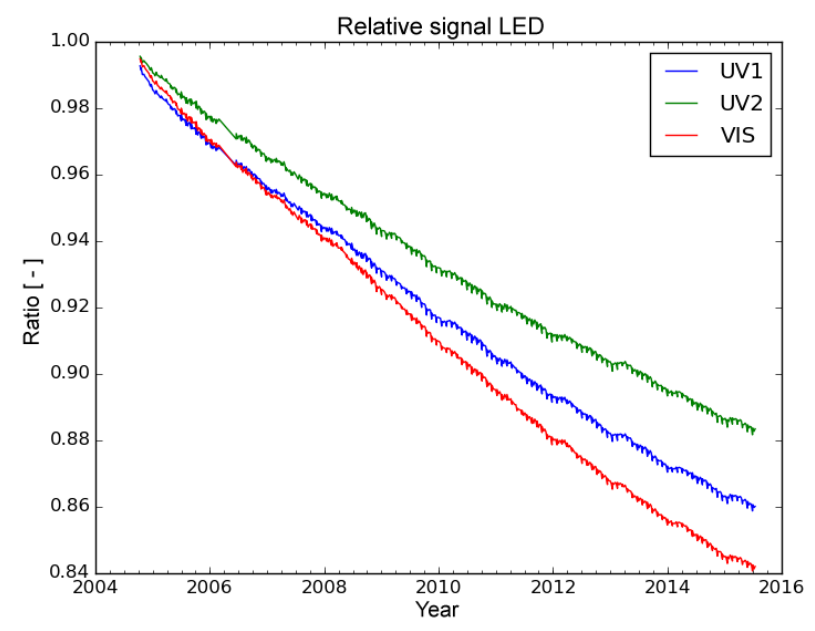

Figure 6. Signal change of LED during the mission. Each data point shows a measurement divided by a reference measurement from the beginning of the mission.

column are stored in the L1B radiance and irradiance output products.

For the second method, the wavelength calibration is performed by fitting a reference solar spectrum (Dobber et al., 2008b), an ozone absorption spectrum and a Ring spectrum to measured radiances. The latter two components are excluded from the irradiance fits. The reference spectrum is divided in 8 windows for the UV1 channel, 18 windows for the UV2 channel and 22 windows for the VIS channel. The fits provide a set of wavelengths that are approximated by a polynomial with the corresponding coefficients (similar to Eq. 1) stored in the L1B calibration product. In the original design of the OMI L1B processor, only the parameters based on the first method were stored in the L1B products. These are the standard wavelength calibration parameters predominantly used by Level 2 developers. Later in the mission the wavelength fit parameters were also recorded in the L1B calibration product. Users are advised to implement the wavelength parameters of the first method. Expert users may also benefit from the wavelength fit method, once they find that these parameters are more suitable for a particular L2 application.

\section{Basic instrument performance}

The following chapter describes the basic performance of the OMI instrument during 12 years of flight. The basic performance of OMI was monitored using the Trend Monitoring and Calibration Facility (TMCF, 2006). We also developed various trending tools supplementing and extending the basic TMCF metrics.

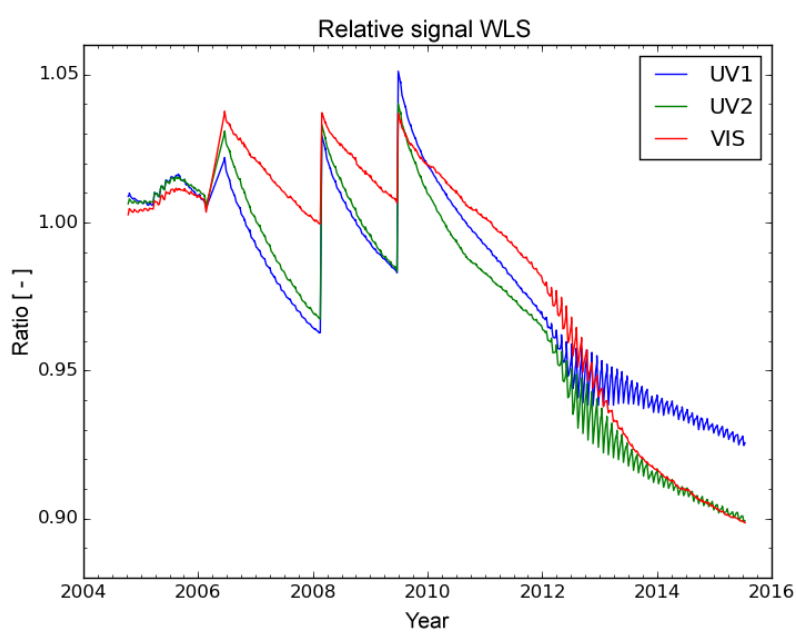

Figure 7. Signal change of WLS during the mission. Each data point shows a measurement divided by a reference measurement from the beginning of the mission. The three abrupt throughput changes in 2006-2009 are caused by the long (14 min each) WLS duty cycles.

\subsection{Monitoring calibration light sources}

Calibration measurements with the LED are performed once per day and with the WLS once per week. In the analysis of this data the average signal in the OMI channel (UV1, UV2 or VIS) is divided by a reference signal, which is an average signal of that channel at the beginning of the mission. The detected long-term changes in the calibration light sources are summarized in Figs. 6 and 7. These are summaries of the overall changes of the calibration pathway throughput; therefore, it is not possible to distinguish between the degradation caused by the light sources, the optical elements, the detector or the electronic components. The WLS (Fig. 7) shows three abrupt changes in years 2006, 2008 and 2009. This source is used once a week, usually being switched on only for a short duration. So far, the WLS was activated 3 times for about $14 \mathrm{~min}$ (cf. the routine $1 \mathrm{~min}$ long calibration cycles). Such long duty cycle causes a temperature spike inside the WLS bulb, making the halogen cycle more effective. During these events the intervening tungsten depositories are removed from the inner surface of the bulb, thus increasing the WLS output. There is no explanation for the erratic WLS behavior starting in 2012.

The main purpose of the LEDs is to monitor linearity and CCD detector properties. The observed $15 \%$ decrease in the LED output over the mission time does not impede the calibration routines. The main purpose of the WLS is a monitoring of the CCD detector properties, pixel response nonuniformity (PRNU) inclusive. This source is not used for radiometric calibration. An overall long-term decrease of $10 \%$ in the lamp output as well as the three abrupt increases of the 
Table 3. Electronic gain values for different channels and spectral bands.

\begin{tabular}{lrrr}
\hline Channel & $\begin{array}{r}\lambda \min \\
(\mathrm{nm})\end{array}$ & $\begin{array}{r}\lambda \max \\
(\mathrm{nm})\end{array}$ & $\begin{array}{r}\text { Gain } \\
\text { value }\end{array}$ \\
\hline UV1 & 264 & 286.2 & 40 \\
& 286.2 & 301.5 & 10 \\
& 301.5 & 311 & 1 \\
UV2 & 307 & 383 & 1 \\
VIS & 349 & 358.8 & 4 \\
& 358.8 & 504 & 1 \\
\hline
\end{tabular}

lamp output do not pose any problems for the relevant CCD characterization.

\subsection{Detector degradation}

The OMI CCD detector is proven to be sensitive to cosmic radiation, despite the $\sim 10 \mathrm{~kg}$ of aluminum shielding (Dobber et al., 2006) added to the instrument preflight. While in orbit, the cosmic radiation has three effects: very short-duration spikes, changes in dark current, and an increase in the numbers of pixels affected by random telegraph signal (RTS) changes (see below). These three effects are accounted for by the ground-processing software. The short duration peaks are corrected with a transient outlier correction algorithm. The increase in RTS pixels is detected, and a flagging scheme is updated accordingly. To correct for an increase in dark current, background measurements are performed at the night side of every orbit. There is a full-cycle analysis with quality control of these background measurements in the TMCF that results in a daily update of the time-dependent operational parameter file (OPF) used in the L1B processor. The parameters that have the most impact on Level 2 data retrieval and parameters demonstrating long-term instrument stability are discussed in the following subsections.

\subsubsection{Detector electronics: gain ratios and electronic offsets}

The measured spectrum that comes out of the CCD detector is amplified by the electronics unit. Different parts of the measured spectrum are assigned different electronic gains, thus substantially improving the data quality at the wavelength affected by strong ozone absorption (UV1 and UV2 range in particular). The high-gain parts will then have less readout noise and quantization noise. Both CCD detectors can be divided in four different areas, each with its own gain setting. The gain values for the different channels and spectral bands are shown in Table 3. Absolute gains cannot be measured during the mission but relative gains (the gain ratios) can. The gain ratios are calculated out of a series of LED measurements. Once per month 10 LED measurements are recorded for all four gains, immediately followed by a series of 10 LED measurements with the gain G1 (gain factor equals 1). These 10-exposure series are averaged and then normalized by the G1 average. Then the four areas in the resulting image with different relative gain values are averaged. This results in four relative gain values. The ratio of the measurements with gain setting G1 should be exactly one. In the top left panel of Fig. 8 we see that the ratio is 1.0002 for the VIS channel. This is an indication for the accuracy of the analysis method.

The variations in gain values are not corrected by the L1B processor, hence they should be accounted for as multiplicative errors of the output signal. The shown long-term UV gain changes $(\sim 0.05-0.25 \%)$ should not be perceived by the majority of L2 algorithms that are usually sensitive to radiometric errors in excess of $\sim 0.25-0.5 \%$, especially when errors show distinctive wavelength dependence. Such wavelength dependence does not exceed $\sim 0.1-0.15 \%$ in the UV case. The changes are more noticeable $(0.25-0.35 \%)$ in the VIS domain. However, the temporal behavior of the VIS gains is essentially (to $\sim 0.1 \%$ ) the same. Hence, this suggests a negligible impact on the $\mathrm{L} 2$ retrievals.

Every measurement (radiance, irradiance or calibration) has an electronic offset. The electronic offset is added to the signal to prevent negative values in the amplified signal. Each gain setting has a different offset value. The electronic offset is determined from the first readout in a measurement (the readout register). All readout register measurements from the mission are stored in the TMCF. The electronic offsets are evaluated as follows. In a readout register measurement, all pixels with the same gain value are averaged. Since there is no signal in a readout register measurement, this average equals to the electronic offset for a given gain value.

Behavior of all offset values during the mission is shown in Fig. 9. We detect the largest variation around $0.5 \%$. This is accounted for as an additive error and corrected by the L1B processor. Therefore, such changes do not impact Level 2 retrievals. From Fig. 9 it can be seen that the trend in the UV channel differs from the VIS channel. The two channels have individual CCD detectors and supporting electronics, however, of a similar design. Hence, both detectors should show similar temporal behavior. The registered differences remain unexplained. The shown trends are based on standard radiance products, where the gain settings G10 and G40 are not used for the VIS channel. Thus, the VIS data are lacking in the lower panels of Fig. 9.

\subsubsection{Linearity of the CCD output amplifier}

The output amplifier of the CCD can cause significant nonlinearity effects when the incoming signal produces more than $2 \mathrm{e} 5$ electrons (67\% of the pixel full well). All measurements (radiance, irradiance and calibration) are corrected for this non-linearity effect by the L1B processor. If the signal exceeds the 2e5 electrons limit for a certain CCD pixel, a non-linearity flag will be raised for that pixel. The percent- 

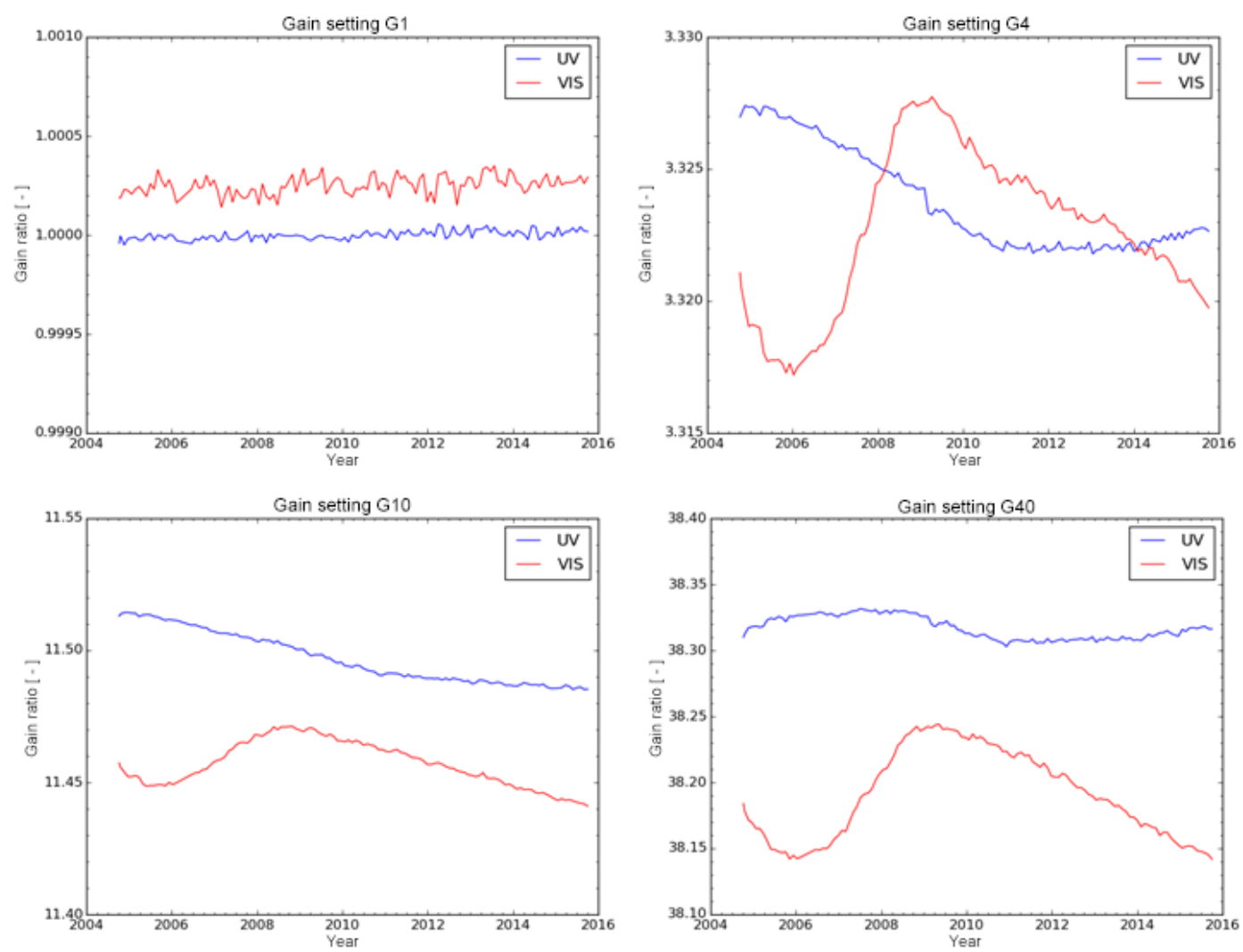

Figure 8. Gain ratio trends over the mission time for the four gain settings. The very small deviation from 1 for gain setting G1 in the VIS channel (upper left panel) is an indication of the accuracy of the analysis method.

ages of pixels with non-linearity flags are shown in Fig. 10. We regard the percentage of flagged pixels as reasonably low. It does not exceed $\sim 0.1$ and $\sim 0.001 \%$ in the VIS and UV channels, respectively, with an overall tendency to gradually diminish on a top of distinctive seasonal cycles for the former and noisy behavior (albeit around a very low level) for the latter.

Once per month linearity measurements with the LED and WLS are performed. For the LED a series of binned measurements with exposure times between 0.1 and $6 \mathrm{~s}$ is done. For the WLS the exposure times are between 0.4 and $1.6 \mathrm{~s}$. Analysis of the data has shown that the WLS measurements are not suitable for non-linearity analysis because the WLS shows too much drift during a measurement, up to $1.4 \%$ where the total non-linearity is expected to be around $3 \%$. The drift of the LED during a measurement is smaller than $0.1 \%$. The linearity analysis results in curves of deviations from linearity vs. register charge. These curves for a number of samples during the mission are shown in Fig. 11. It can be seen that non-linearity does not vary much during the mission.

\subsubsection{Detector dark currents}

The OMI CCD detectors are operated at $T=265 \mathrm{~K}$, controlled to $\pm 10 \mathrm{mK}$. At this temperature the dark current of the CCDs was $85 \mathrm{el} . / \mathrm{pix} \mathrm{s}^{-1}$ for UV and $132 \mathrm{el} . / \mathrm{pix} \mathrm{s}^{-1}$ for VIS at the beginning of the mission. Dark-current measurements are performed on the night side of each orbit, thus revealing the levels of CCD's shot noise. The dark-current level is directly proportional to exposure time, hence the night-side calibration employs various integration times, up to a $136 \mathrm{~s}$ duration. In the analysis we use image-averaged dark-current measurements. These averages are corrected for electronic offset, divided by the integration time and provided for each orbit in the L1B calibration files. As expected, during the mission the average dark current has gradually (Fig. 12) increased to $\sim 800 \mathrm{el} . / \mathrm{pixel} \mathrm{s}^{-1}$. The CCD detector is sensitive to cosmic-ray hits that result in the observed dark-current increase. The GOMOS mission (Kyrölä et al., 2004) has used the same CCD detector. The analysis of dark-current trends of that detector has shown an increase of dark current during the mission (Bertaux et al., 2010) that is even higher compared to OMI. Although seemingly substantial, such increase does not negatively reflect of the L2 data production, as long as the processing pipelines use dark-current measurements taken at each OMI orbit. These measurements are used to 

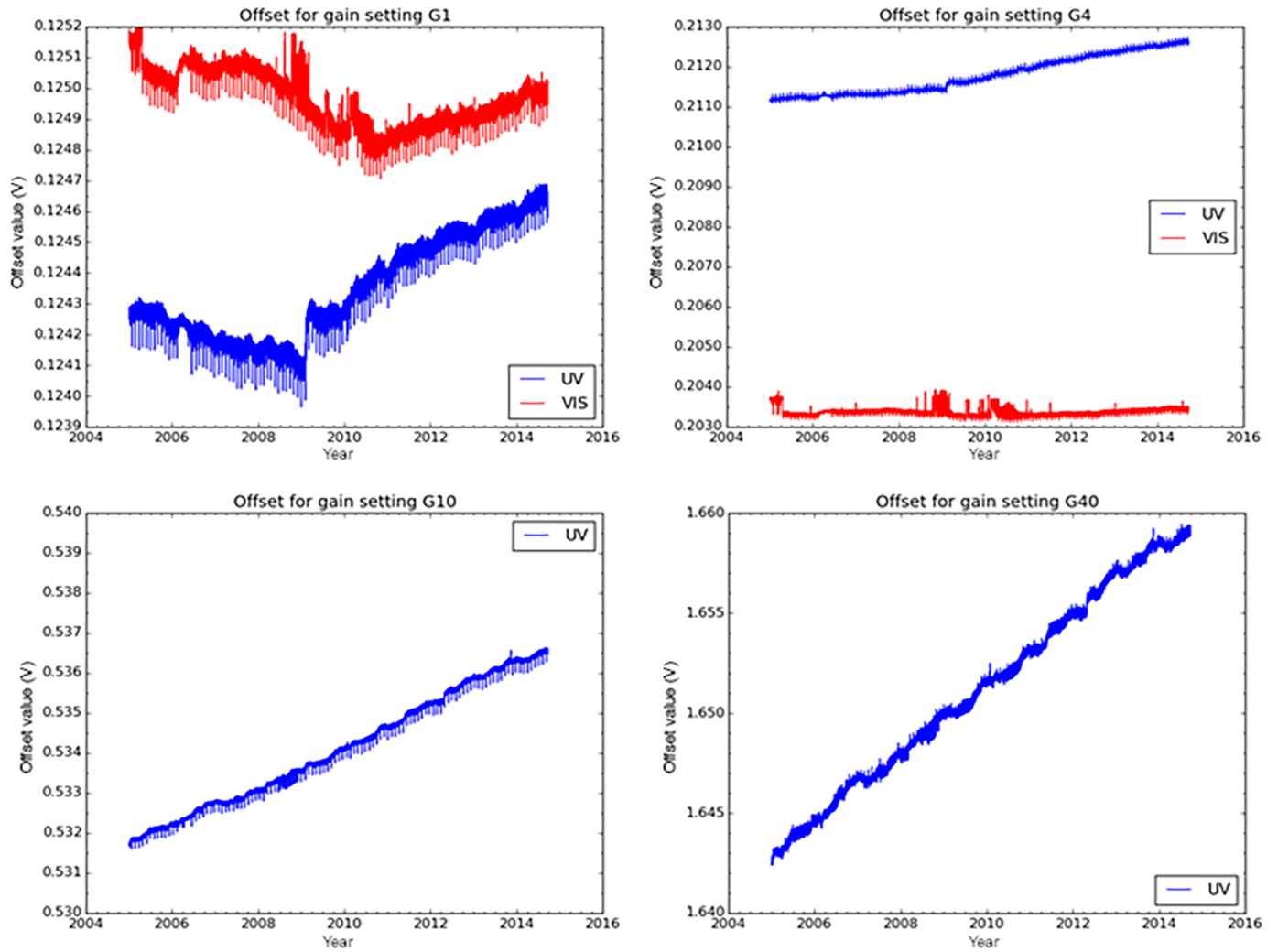

Figure 9. Changes in the electronic offset over the mission time for the four gain settings. Note that offset values for the gain setting G10 and G40 of the VIS channel are not used in radiance measurements.

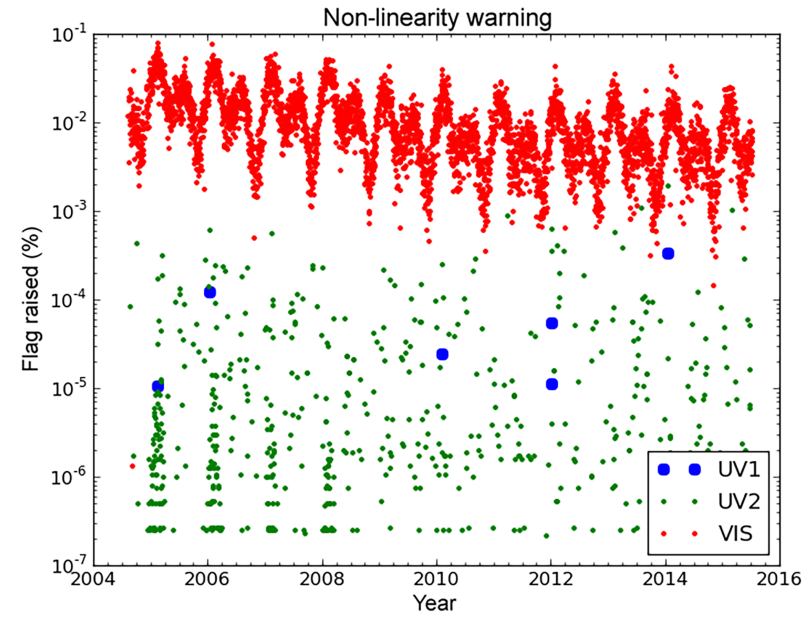

Figure 10. Non-linearity warnings in output of the CCD amplifiers over the mission time.

correct the (ir)radiance measurements from the same orbit, thus effectively removing the dark-current contribution.

The increase in dark current can also be seen in the dark current distribution. Histograms for the UV channel for various years are shown in Fig. 13, along with the UV bad-pixel threshold (see Sect. 4.2.5; when a pixel has a dark-current

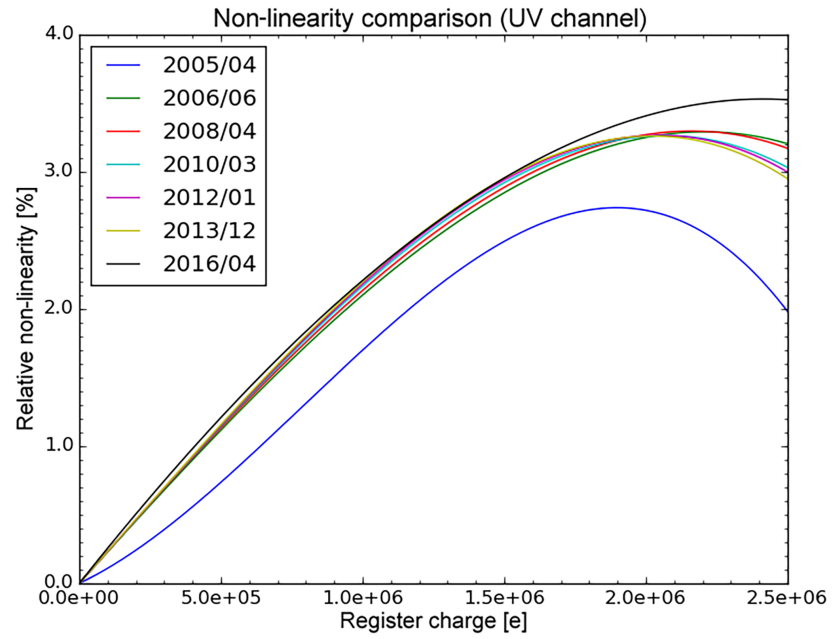

Figure 11. Non-linearity comparison for the UV channel for different years. Apart from the 2005/04 curve, the curves are pretty similar, which indicates that non-linearity has not changed much during the mission. The curves for the VIS channel are similar to the UV channel curves.

value above the pre-set threshold, it is flagged as bad by the L1B processor). The corresponding histograms for the VIS channel look similar. 


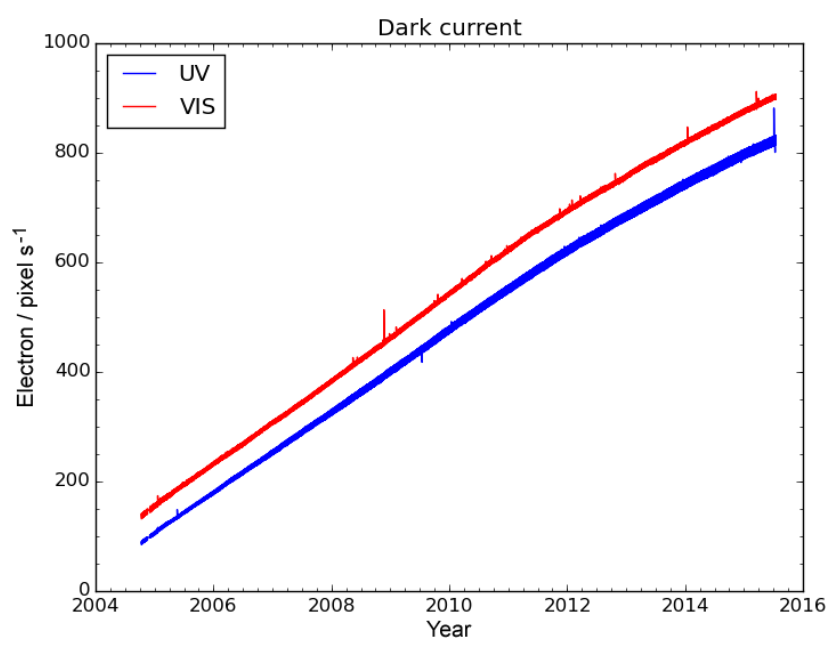

Figure 12. The average dark currents for two CCDs.

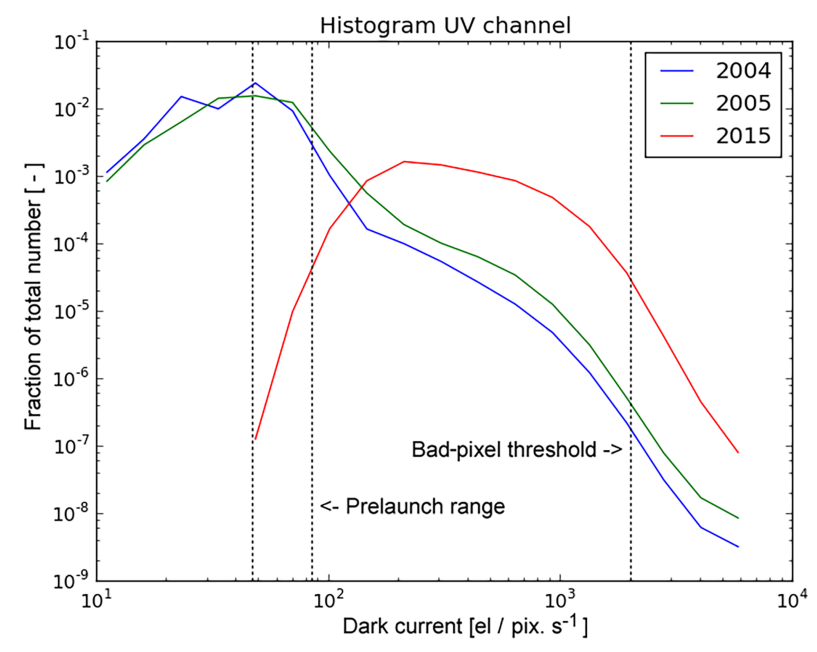

Figure 13. Histograms of dark current measured in the UV channel.

\subsubsection{Random telegraph signal}

A pixel affected by the random telegraph signal has an average dark current that randomly toggles between two or more levels. Hence, for a given pixel its RTS behavior can be deduced via statistical analysis of the corresponding darkcurrent levels. This analysis was done on specific darkcurrent measurements that are performed once a day and employ long integration time in order to improve statistics. There are two different dark-current measurements used in this analysis, with 136 and $2 \mathrm{~s}$ integration times, acquired at the same orbit. The measurement with the short integration time is subtracted from the measurement with the long integration time. For each pixel in the resulting image the dark current is calculated by dividing the signal by the difference in exposure times. A series of measurements is taken, belonging to 60 consecutive days. From this dataset the following statistics are calculated for every pixel: mean, variance, observed to expected variance ratio, skewness and kurtosis. Each statistics has pre-set threshold values. If, for a given pixel, one of the statistics exceeds the threshold, it is flagged as an RTS pixel. Examples of a few RTS pixels and their histograms are shown in Fig. 14. The number of pixels that show RTS behavior has increased from 0.1 to $0.7 \%$ over the mission time (Fig. 15).

The results shown in Fig. 15 are for unbinned pixels. Standard radiance and irradiance measurements are performed by binning eight consecutive rows (cross-track direction). If one or more of the eight original (unbinned) pixels carries the RTS warning flag, and then the binned pixel will also carry the RTS warning. Therefore, in the L1B products the number of RTS flagged pixels is 8 times higher than shown in Fig. 15 .

Despite the increase observed in RTS on the OMI CCD detectors, the short-lived nature of these events appears to limit their overall impact on the most sensitive Level 2 retrievals. For example in the OMI BrO spectral fitting algorithm (Kurosu et al., 2004), the fitting residuals used for diagnostic purposes grow by less than $5 \%$ over the OMI mission. The same applies to the fitting residuals of the OMCLDRR fitting algorithm (Vasilkov et al., 2008).

\subsubsection{Bad-pixel flagging}

Pixels are considered bad if their behavior is perceived as offnominal. This can, for instance, be related to the anomalous (either exceedingly high or low) dark-current readings. Alternatively, bad behavior may be detected via inadequate (either high or low) response to illumination from a calibration source. This is monitored in the TMCF using dark-current, WLS and LED measurements. If values exceed the absolute threshold limits during dark-current measurement, the pixel is flagged as bad or dead. The limit values for flagging were determined in the first years of the mission, in an empirical manner, mostly based on the dark-current values at that time as well as the notion that the number of pixels being flagged should be neither exceedingly high nor too low. The upper limit value for a bad pixel is $2000 \mathrm{el} . / \mathrm{pix} \mathrm{s}^{-1}$ and for a dead pixel is $3000 \mathrm{el} . / \mathrm{pix} \mathrm{s}^{-1}$. The lower-limit values were set to $1 \mathrm{el} . / \mathrm{pix} \mathrm{s}^{-1}$. These values have not been changed during the mission. Since the dark-current values steadily rise, so does the numbers of flagged pixels. By definition, a dead pixel does not necessarily have zero response to incoming light. It can also be called a hot pixel, because the dark current of the pixel is much higher than expected. If a pixel signal deviates too much from the average pixel value in a WLS or LED image, it is also flagged as bad or dead. The result of this analysis is a bad and dead pixel map, which is recorded in the operational parameter file for further use in the L1B processor. The predominant percentage of bad-pixel flags is related to the pixels surpassing the pre-set dark-current badpixel threshold value of $2000 \mathrm{el}$./pix s ${ }^{-1}$. The bad-pixel percentages for the UV and VIS channels are shown in Fig. 16. 
(a)
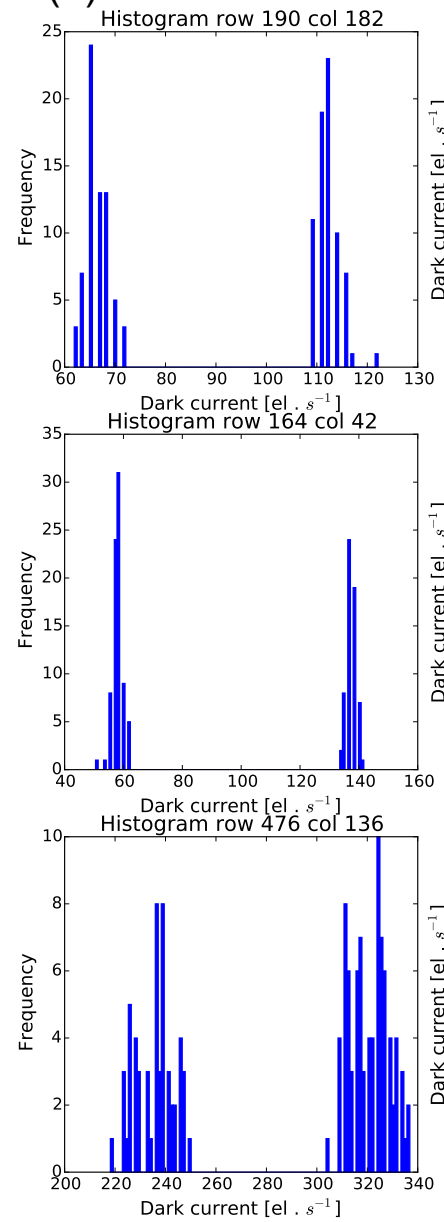

(b)
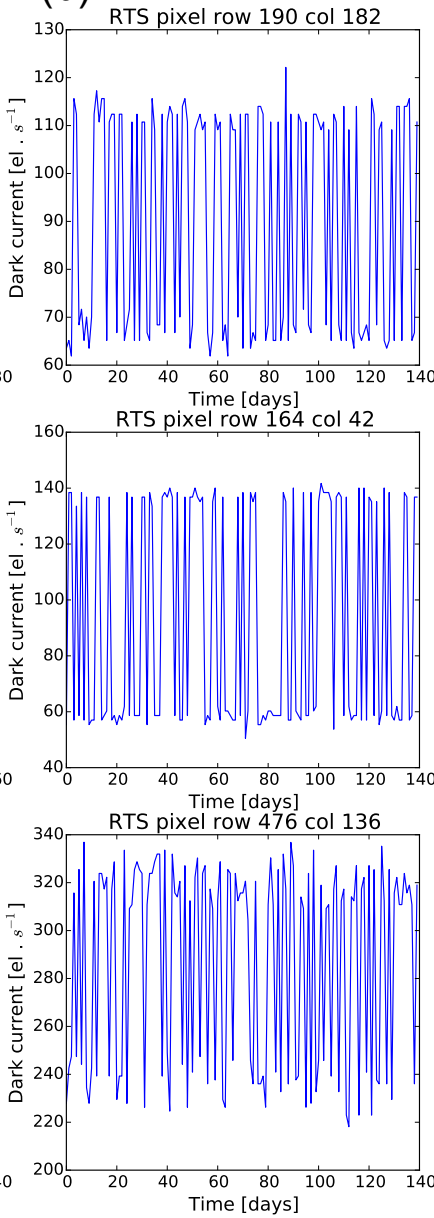

(c)

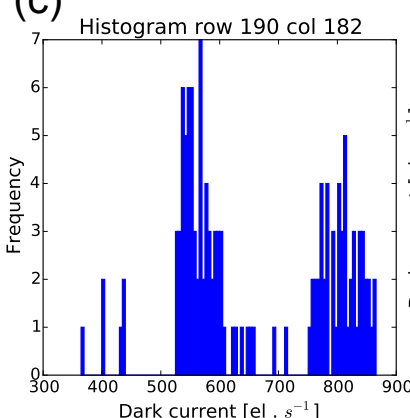

(d)
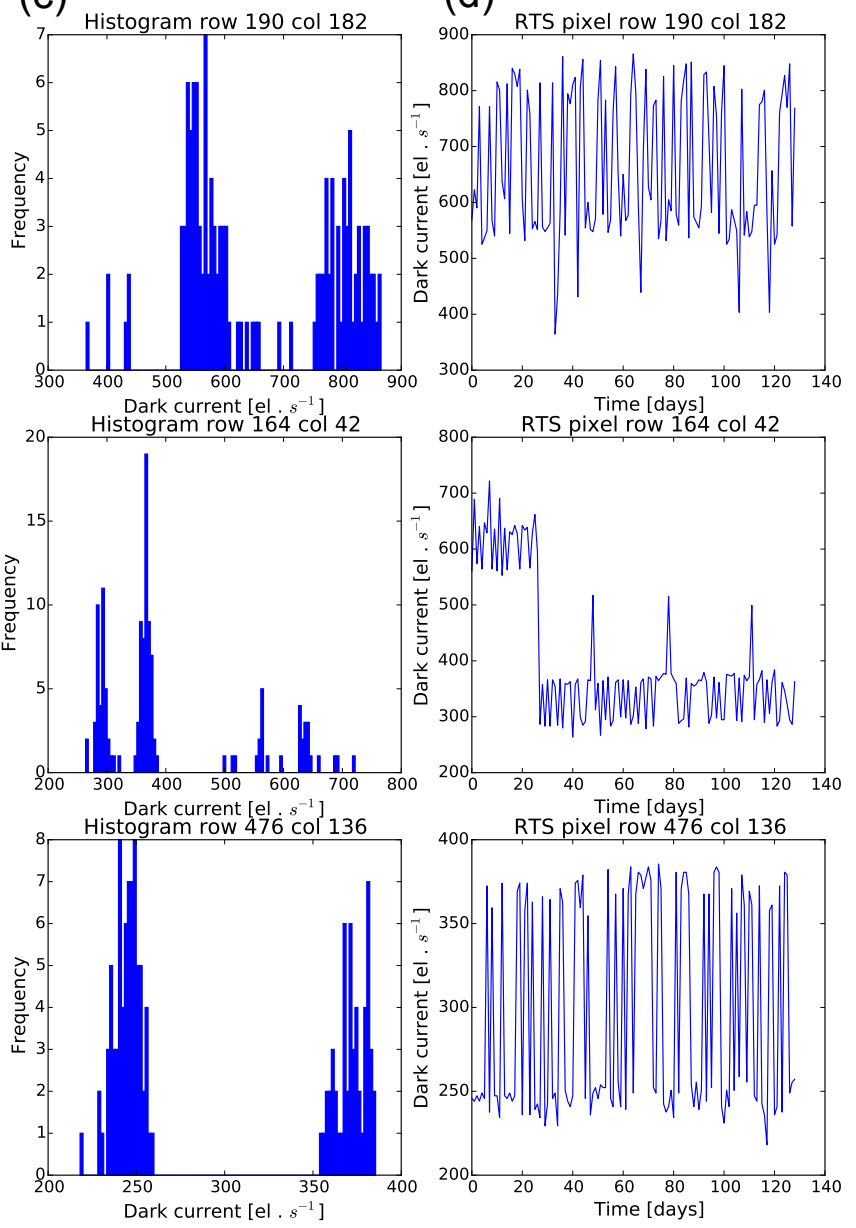

Figure 14. Examples of RTS pixels. The left two columns show results for 2008, and the right two columns show results for 2015. (a) and (c) show histograms of RTS pixels. (b) and (d) show dark-current evolution over time. In 2015 the dark current and noise are higher, which gives the histograms a smoother character. Note the different scales that are used for 2008 and 2015.

The trends in Fig. 16 should be considered in conjunction with the results from Figs. 12 and 13. The dark current of the detectors is increasing (Fig. 12), hence a larger number of pixels is flagged as bad. As in Fig. 15, Fig. 16 shows the unbinned pixels. Therefore, due to binning the amount of the flagged bad pixels in the L1B products is 8 times higher than shown in Fig. 16.

\subsubsection{Signal-to-noise ratio}

The OMI instrument lacks precisely calibrated, sufficiently stable onboard sources. This makes direct estimation of the signal-to-noise ratio from radiances a very challenging task that is further complicated by earthshine variability stemming from ever-changing geophysical factors. Hence, in order to reveal SNR trends, we revert to the values provided by OMI irradiances as well as specific OMI science products derived from irradiances.
Each daily solar measurement comprises 77 individual exposures taken within a fairly narrow range of relative (to QVD) solar elevations. We use these measurements for SNR estimates by choosing all the data within a $\pm 3^{\circ}$ elevation range. For each wavelength step in each exposure and each FOV (row), we calculate the difference between the measured irradiance and the average of irradiances from adjacent wavelengths. Then the RMS values of these differences are binned over 11 wavelength steps and averaged over all rows. The resulting SNRs are shown in Fig. 17, where full and dotted lines follow the monthly averages for January 2005 and 2016, respectively. The small but systematic SNR decline goes in line with the steady growth of RTS events.

Some of the science products turn out to be more sensitive to the gradual SNR decrease. As an example, Fig. 18 shows time-binned (yearly) RMS of the ratios of the solar indices measured in the VIS (Ca II lines) and UV1 (Mg II line) irradiances measured by OMI (Deland and Marchenko, 2013) 


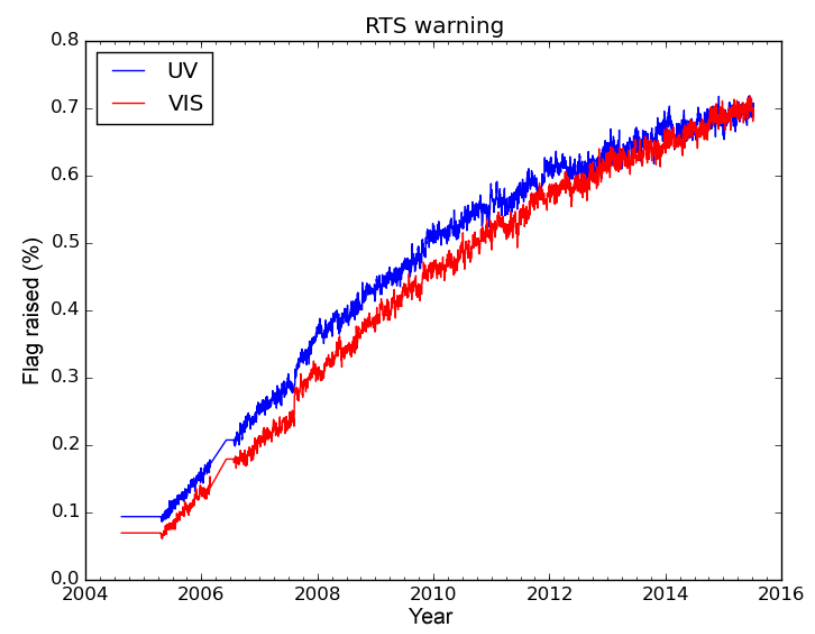

Figure 15. Random telegraph signal (RTS) flagging trend over the mission. These results are for unbinned pixels. The binned L1B pixels have flagging rates that are 8 times higher.

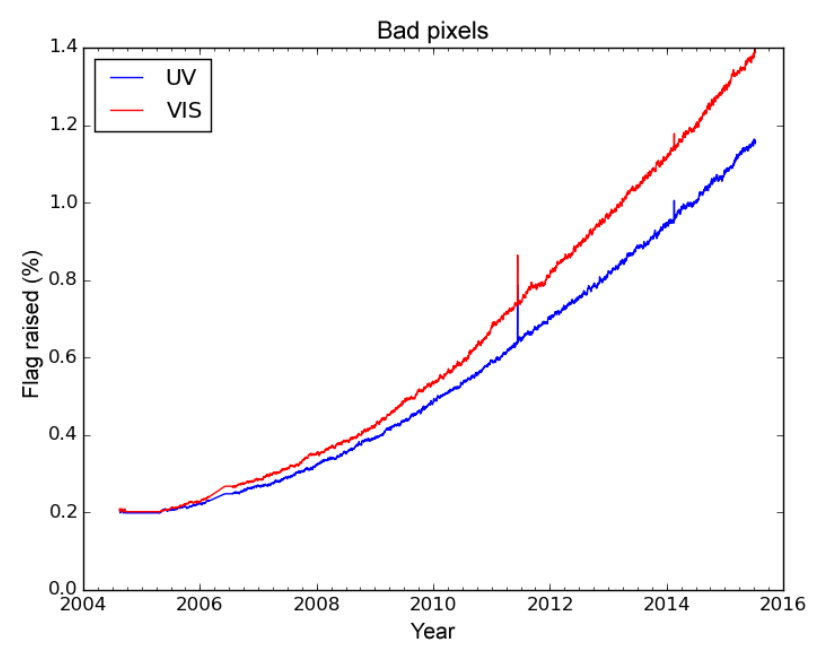

Figure 16. Bad-pixel flagging over the mission time for unbinned pixels. The binned L1B pixels have an 8 times higher flagging rate.

and the SOlar Radiation and Climate Experiment (SORCE; Snow et al., 2005) on a daily basis. For a particular spectral line (usually, the prominent absorption lines, such as $\mathrm{Mg}$ II at $280 \mathrm{~nm}$ or H and K Ca II doublet at 393 and $397 \mathrm{~nm}$, respectively), the solar index is defined (see more details in Deland and Marchenko, 2013) as a ratio between the solar flux at the line core to the average solar flux measured at the line's wings. Such indices serve as very sensitive indicators of solar activity, with the lines in question, $\mathrm{Mg}$ II and Ca II, changing in almost perfect agreement, however, with the line-profile variability in the Ca II lines being, on average, 5-7 times lower than the relative changes in the $\mathrm{Mg}$ II lines. Inspection of the solar Mg II indices provided by OMI and, independently, by SORCE shows no discernible time-dependent trends in the relative noise level (Deland and

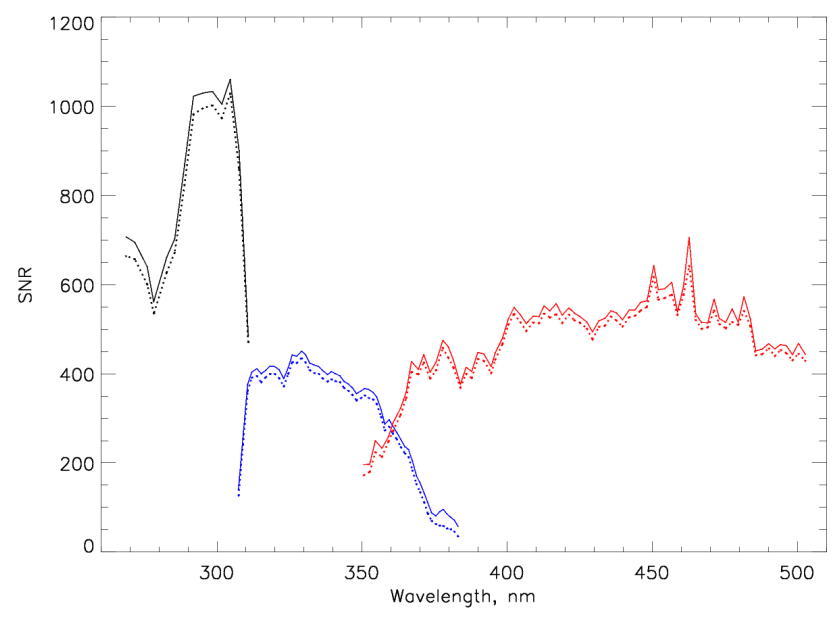

Figure 17. The wavelength- and time-binned solar irradiance SNRs for January 2005 (full lines) and January 2016 (dots) in UV1 (black), UV2 (blue) and VIS (red) channels.

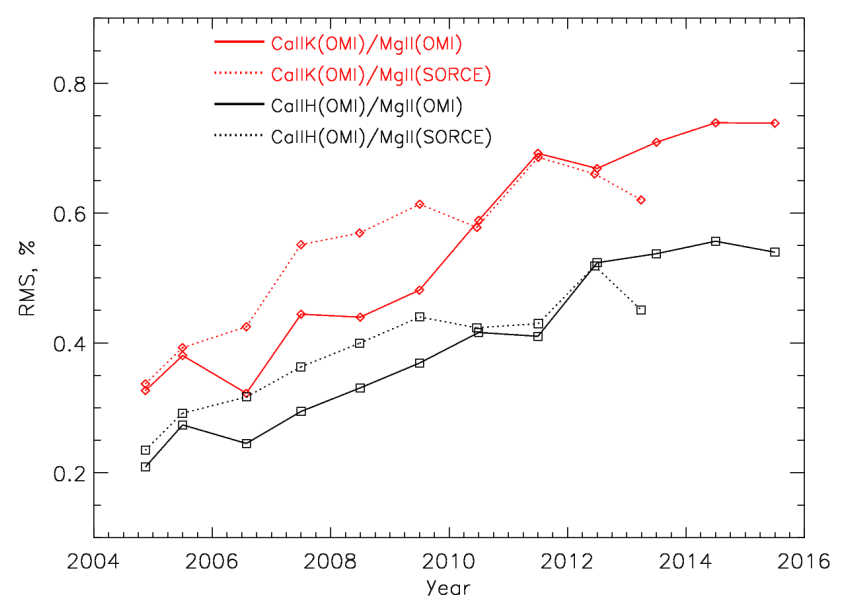

Figure 18. Time-binned (yearly) RMS of the ratios of the solar indices derived from the VIS and UV1 data.

Marchenko, 2013). This is not surprising, considering the relatively small UV1 SNR changes seen in Fig. 17. Hence, we regard the $\mathrm{Mg}$ II values as a relatively noise-free baseline and conclude that the gradual growth of RMS in the ratio of the $\mathrm{Ca}$ II and Mg II indices is caused by a steadily increasing instrument noise, with OMI Ca II data being highly susceptible to these changes.

\subsubsection{Pixel response non-uniformity}

Individual CCD pixels respond differently to incoming light. This is a detector property that depends on wavelength. This pixel response non-uniformity is about $5 \%$ for wavelengths around $270 \mathrm{~nm}$ and decreases to $0.1 \%$ for wavelengths around $500 \mathrm{~nm}$. If PRNU is not corrected properly, it will cause high-frequency structures in the calibrated L1B output products. To determine PRNU a white light source 


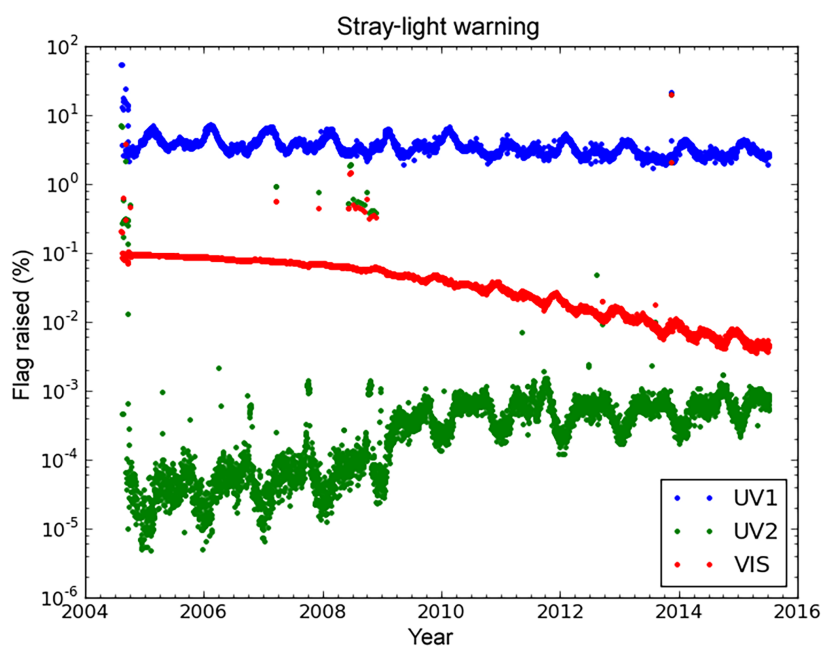

Figure 19. Stray-light warning trend over the mission.

with a high spectral stability (up to $10^{-4}$ ) is needed. In practice it is difficult to obtain such a light source, both onground and in flight. On the ground the PRNU was determined by illuminating the QVD diffuser, because this diffuser introduces the smallest features (around $10^{-4}$ ). In flight the only option is to use the WLS light that passes through the transmission diffuser, which introduces features on a $1 \%$ scale. The transmission diffuser feature pattern was determined by comparing an on-ground measurement with the QVD diffuser and an on-ground measurement with the WLS light through the transmission diffuser (Dobber et al., 2006). Nevertheless, it turned out to be very difficult to correct inflight WLS measurements for the transmission diffuser features. The WLS is degrading in flight, and the dark current and noise of the CCD detector are increasing. Therefore, the PRNU evolution during the mission has not been monitored. The PRNU correction in the L1B processor is currently still based on the results of the on-ground calibration.

\subsection{Stray light}

Various OMI L2 products show different sensitivity to the stray-light contamination, depending on how the OMI radiances and irradiances are combined in a specific product. Below we show that in most cases any long-term trends that may be ascribed to gradual changes in the stray-light levels do not exceed the achieved $\sim 0.5-1.0 \%$ detectability limits. One should note, however, that some spectral domains show enhanced sensitivity to the stray-light contamination: e.g., $\lambda<290 \mathrm{~nm}$ in UV1 and $\lambda<320 \mathrm{~nm}$ in UV2, both spectral ranges affected by the strong $\mathrm{O}_{3}$ absorption, thus resulting in relatively low radiance readings and, as a consequence, a high sensitivity to the additive stray-light component. Such sensitivity is augmented by the distinct wavelength dependence of the stray-light contamination that may not be completely captured by the implemented correction algorithm: e.g., UV1 irradiances - see below. Science products that derive information from the discrete intensity or ratio of reflectances are more sensitive to stray-light errors, while the differential optical absorption spectroscopy (DOAS), spectral fitting and principal components analysis (PCA) algorithms are relatively insensitive to stray light.

Based on outcome of the preflight tests, the stray-light contamination is modeled in the L1B processor assuming a smooth (low-order polynomial) behavior in the spatial and spectral dimensions. The spatial stray light is measured at the dedicated stray-light rows right below or above the imaging area of each CCD detector: the USA and LSA CCD areas in Fig. 4. The signals from these rows are linearly interpolated over the entire CCD image. The spectral straylight dependence is evaluated at specific CCD columns, then interpolated at all wavelengths of a channel (UV1, UV2 or VIS) and whenever applicable extrapolated to the wavelengths of another channel. The spatial (row-wise) and spectral (column-wise) stray-light components are combined to form a complete stray-light envelope, which is eventually subtracted from the image. If the stray-light signal is too large (i.e, the corrected radiances turn negative), flags are raised for the corresponding parts of the image. The results of such stray-light flagging are shown in Fig. 19. For the UV1 channel, the one with the highest stray-light warning level (low radiances in the ozone-absorbing domain), there is no significant increase in warnings over the mission time. Considering the potential influence of the row anomaly (see Sect. 5 for more details) on the stray-light estimates, one may conclude that the currently implemented stray-light correction algorithm adequately accommodates such changes in UV1. On the other hand, despite the relatively low level of the flagged events, the VIS channel shows some RA sensitivity: note the rapid increase in flagging at the beginning of 2009 coincident with a major RA event.

Since the described procedure of stray-light removal uses preflight characterization along with some general assumptions about the spatial and spectral stray-light behavior, there is a need for independent estimates of the stray-light contamination. This could be done relying on the Sun as a relatively stable and predictable light source. In order to follow changes in the wavelength registration, as well as spectral line-profile shapes, we select multiple well-developed, relatively deep absorption features (usually, blends of the solar absorption lines) spanning the UV1, UV2 and VIS ranges. In the daily irradiances and in every earthshine spectrum we measure wavelength centroids of the absorption lines, full widths at half maxima and line depths. The line depth and FWHM are related to the radiances coming from the fixedwavelength, relatively line-free spectral regions in the immediate vicinity of the measured absorption.

Line-profile estimates from the daily irradiance measurements are checked for $\pm 2 \sigma$ outliers and binned into 3-month averages. In the earthshine data, at each orbit and each FOV, the measurements are checked for $\pm 2 \sigma$ outliers and averaged 


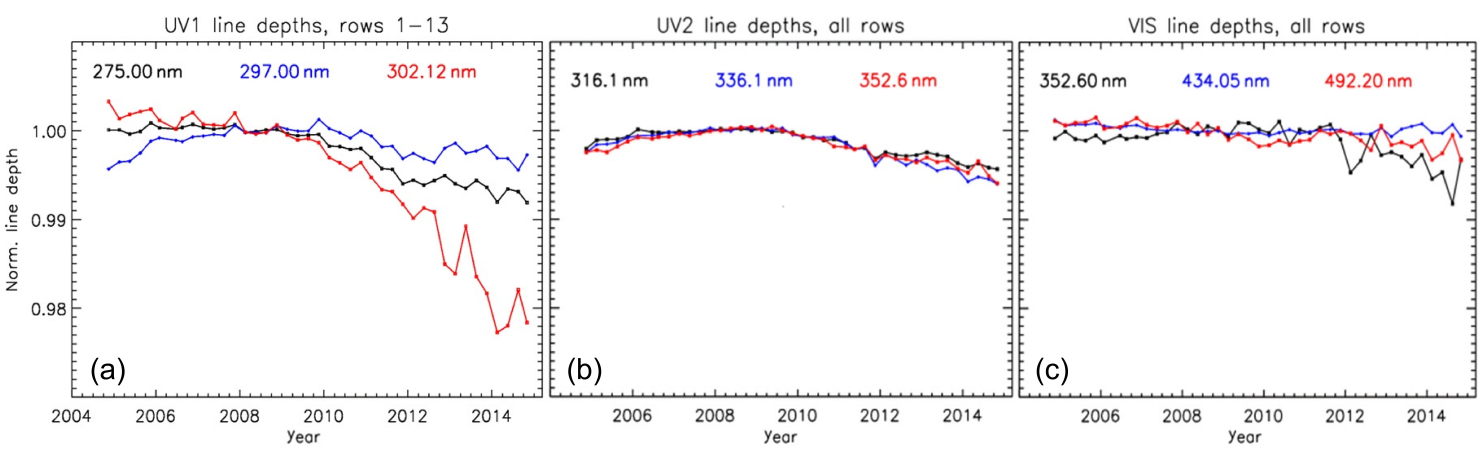

Figure 20. Changes in the normalized line depths of prominent absorption blends in the solar irradiances observed in UV1 (a), UV2 (b) and VIS (c).

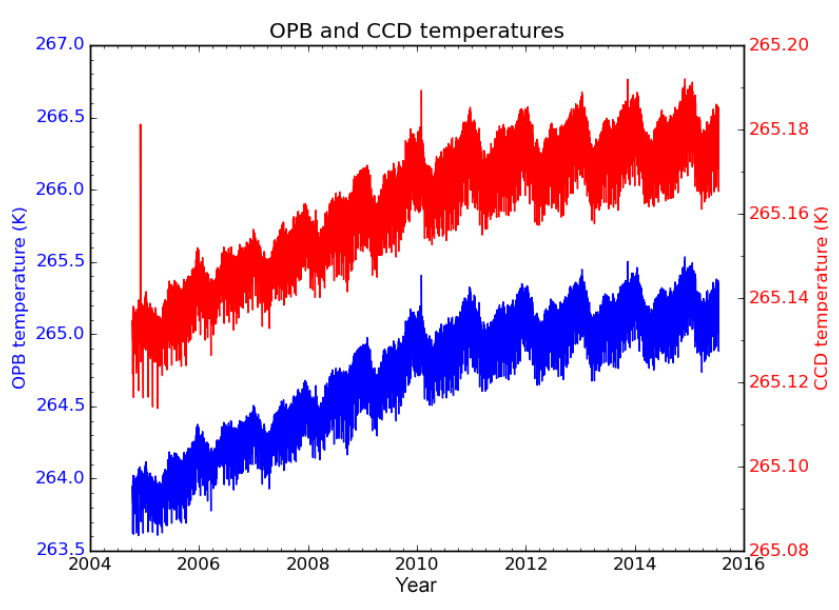

Figure 21. Changes in the OPB and the UV channel CCD temperatures.

within 100-exposure orbital blocks. These orbital blocks are assembled into daily means. For each spectral line, each FOV and for a given range of solar zenith angles (SZA; the SZA bins are defined with $10^{\circ}$ increments), the relatively RA-free measurements between 2004 and mid-2008 are used to estimate biannual seasonal variability patterns and subtract them from the individual measurements. The de-trended values are checked for outliers and binned into 3-month averages.

To produce the trends shown in Fig. 20, we combine all the UV2 and VIS rows, and rows 1-13 from UV1 (reasons for the latter are discussed below), bin the values over 3 consecutive months, and then normalize the line depth by the average line-depths values observed during the latest solar minimum (March 2007-August 2009). Both the line depths and, to a lesser extent, FWHMs (not shown) of the absorption blends follow the predictable changes related to the solar cycle (see more details in Marchenko and Deland, 2014). In essence, practically all absorption lines in the OMI irradiances are getting progressively shallower with the gradually (years) increasing solar activity levels. This creates the inverted-U shapes seen in Fig. 20. The long-term changes are far more pronounced in the UV1 range compared to VIS, in line with the expected solar cycle behavior. If there are any instrumental trends, then at this point they cannot be clearly disentangled from the anticipated solar-related changes in the UV2 and VIS ranges. The relative changes (i.e., the deviations from the expected inverted-U shape) in the UV1 line depths point to possible $\sim 0.5-1.0 \%$ instrumental trends, especially considering the temporal behavior of the $302.12 \mathrm{~nm}$ blend, to be compared to the UV2 lines closely following the expected transformations.

We also performed (not shown here) line-depth measurements for various spectral features in the UV2 range of the earthshine spectra. In general, the earthshine trends conform to the inverted-U shapes seen in the UV2 irradiances (the middle panel in Fig. 20); i.e., in radiances the gradual linedepth changes are also mostly driven by the long-term (years) solar variability. However, we noticed some subtle deviations from the expected trends, most likely related to gradual straylight changes not properly accounted for by the currently adopted (Collection 3) approach. Considering the measured line-depth values, as well as magnitudes of the deviations, we assume that such deviations may be caused by $\sim 0.5 \%$ longterm changes in the UV2 stray-light levels, in line with the relative $\sim 0.5-1 \%$ difference in the long-term trends derived from the low-reflectivity and high-reflectivity subsets of the UV2 radiances (see below).

\subsection{Instrument temperatures}

The temperature of the optical bench impacts the wavelength registration. The design of the optical bench is such that thermal fluctuations of the optical bench should have a minimal effect on wavelength registration. In the OMI case there is a small, however, detectable relation between the two quantities. The small seasonal variability in the trend in wavelength registration (see Sect. 6.3) can be directly related to temperature fluctuations. Besides, the dark-current readings depend on temperature. In general, when the temperature of the detector rises by $10^{\circ}$, the average dark current doubles. This 

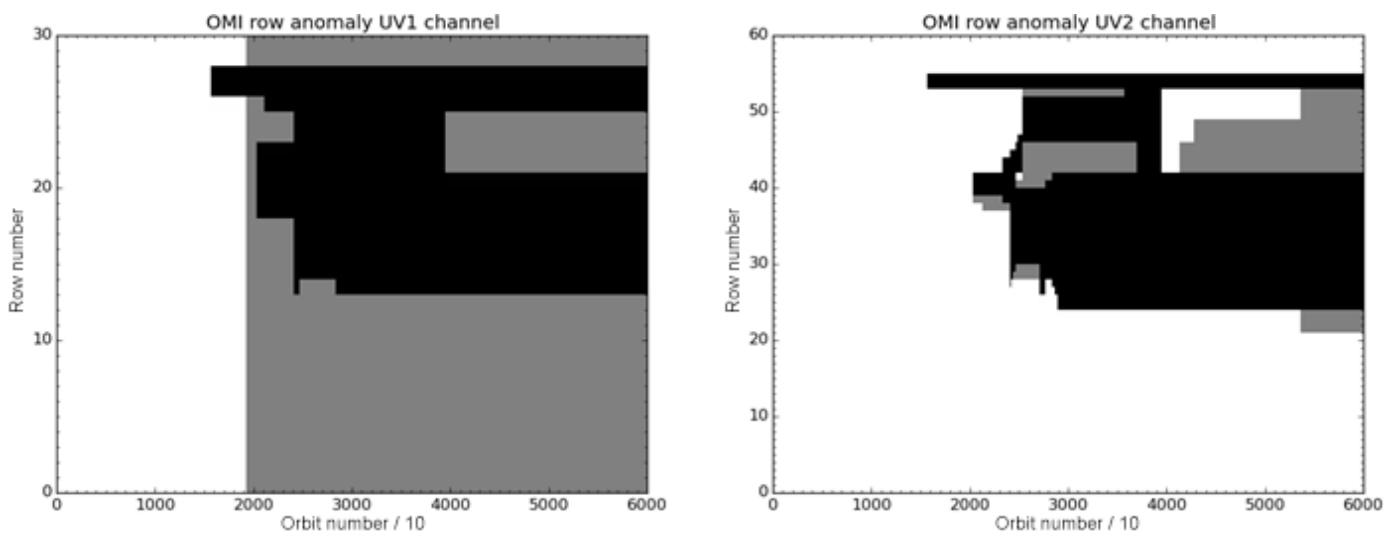

Figure 22. Row-anomaly evolution for the UV1 and UV2 channels. Black areas show full RA-affected orbits, and gray areas mark partial orbits (northern part). The VIS channel looks similar to the UV2 channel.

calls for low and very stable operational temperatures of a CCD detector.

The OMI optical bench is cooled by a passive radiator plate. Without additional heating the temperature of the OPB would be about $255 \mathrm{~K}$. Passive heaters warm the OPB to the operational temperature of $264 \mathrm{~K}$. The temperature of the CCDs is controlled with active heaters in a closed-loop feedback system. The operational temperature is $265 \mathrm{~K}$, controllable to $\pm 10 \mathrm{mK}$. Figure 21 shows the trend in the temperature of the OPB and the UV CCD detector. The trend in the temperature of the VIS channel looks similar to that of the UV channel, with a steady increase of the temperatures early in the mission, which flattens after 2010. The trend in CCD temperature very closely follows that of the OPB, albeit the absolute change is far smaller (note the different scales for $\mathrm{CCD}$ and OPB temperatures). The temperature of the OPB can be controlled with a $1^{\circ}$ tolerance limit. The temperature setting was not changed during the mission. Therefore, the $1^{\circ}$ increase early in the mission is large and should have triggered system's response. Consecutively, the temperature of the CCD has increased by $40 \mathrm{mK}$, thus far exceeding the tolerances of the controlling system. The lack of timely adjustments in both controlling systems remains unexplained. This, however, does not impact the OMI performance in any major way (see below).

\subsection{Voltages}

The electronics unit monitors a number of internal voltage values. Analysis of the voltage data has shown that fluctuations over the mission are small. The largest fluctuation that was seen in the 5 and 12 volt lines was $0.07 \%$. There are two voltage parameters that show larger variability. The WLS voltage shows changes up to $0.5 \%$, and the test voltage changes by $12 \%$. Since the test voltage is not used for nominal operations, this increase poses no problem for radiances, irradiances and Level 2 product retrieval.

\section{Anomalous behavior}

Since June 2007 (the currently accepted date; there are some, though very limited, indications of an even earlier onset of the anomaly) OMI has suffered from the so-called rowanomaly (RA) phenomenon. In this anomaly certain Earthobserving cross-track FOVs (rows) are seemingly blocked, resulting in abnormally low radiance readings. The most probable cause of blocking is a partial external obscuration of the radiance port by a piece of loose multi-layer insulation (MLI) of the instrument itself, but this is not certain. The first signs of the anomaly were detected in rows 54 and 55 (1-based). These rows remain affected ever since. Since May 2008 the anomaly affects image rows $38-42$ (see Fig. 22). At the time of writing the anomaly was relatively stable, permanently affecting UV2 rows $25-42$ and 54-55, and occasionally spreading to rows $43-53$. Figure 22 depicts the RA evolution in the UV1 and UV2 channels. The VIS behavior is somewhat similar to UV2, however, showing different degrees of involvement for the rows in the immediate vicinity to the main RA domain (defined as rows 25-42). The row anomaly affects the data in four different ways:

Blockage effect. Several rows (cross-track viewing angles) have a decrease in signal strength. This decrease is assumed to be caused by something blocking the nadir port of OMI. The blockage effect is a multiplicative, wavelength-dependent factor.

Solar light contamination. Several rows show increased signal level. This increase predominantly happens in the northern part of the orbit, in apparent relevance to the incident sunlight. It is assumed that something outside the nadir port is reflecting sunlight into the instrument. This could be a piece of loose MLI. This increase in signal level has an additive, wavelength-dependent effect.

Wavelength shift. The partial blocking of the nadir port results in inhomogeneous illumination of the OMI spec- 
tral slit. This causes a slight change in the instrument spectral response function, changing wavelength registration.

\section{Earth radiance from outside nominal field of view.}

Several rows may show increased signal levels at certain parts of orbit. This is caused by the earthshine from outside of the nominal FOV reflected into the nadir port. This is an additive factor with time- and FOV-dependent terms, and thus it is the most elusive row-anomaly effect.

Based on Level 1 data, a daily automatic analysis distinguishes between these four row-anomaly effects. A warning flagging scheme is based on the multitude of parameters provided by such analysis. The most influential contributing factors are the number of negative reflectances, the number of overly large reflectances, the reflectance histogram, the mean-scaled wavelength shift, and the wavelength fit failure count. This flagging scheme is added to the Level 1B product. If the daily analysis results shows significant shortterm changes, the flagging scheme is adapted manually. The number of the affected rows has increased since the first appearance of the row anomaly in 2007. Figure 22 shows the affected UV1 and UV2 rows. The VIS channel looks similar to the UV2 channel.

The RA effects grow progressively larger, with pronounced seasonal modulation, in the northern parts of the OMI orbits, when sunlight is coupled into the instrument via the radiance port. Table 3 shows the percentage of rows that is affected for all orbit phases and for the northern parts of orbits, with a noticeable $100 \%$ involvement of the UV1 channel. The row-anomaly effect is not corrected by the L1B processor. The RA flags are included in the affected L1B.

We also performed an independent analysis of the OMI radiances, applying the following procedure. The typical OMI orbit provides $\sim 16402$-second exposures. These are binned into 50-exposure blocks. This initial binning assures better SNR for the spectra obtained over areas with low surface reflectivities and/or high solar zenith angles. Besides, the relatively small size of the bin keeps the gradual orbital drifts in the wavelength registration (thermal flexure) well below $\sim 0.005 \mathrm{~nm}$ for exposures within the bin. For the UV2 data, observed scenes are partitioned into three reflectivity categories: $r<15 \%, 15 \% \leq r \leq 60 \%$, and $r>60 \%$. Such partitioning provides roughly (by a factor of a few) comparable sample sizes. The scene reflectivities at $\lambda=331$ and $360 \mathrm{~nm}$ are estimated by the OMTO3 (total ozone) algorithm. The 50-exposure averages for each reflectivity category are corrected (normalized) for changes related to variable solar zenith angles and then interpolated to a common wavelength grid. The data in each 50-exposure block are wavelengthbinned ( $\sim 0.5 \mathrm{~nm}$ bins) around the relatively line-free spectral areas and then assembled into multiple-day averages (currently, in 15-day blocks; for illustration purposes, 90-day averages are shown in some plots). For each FOV (row), each orbital 50-exposure block and each binned wavelength, we derive the biannual "climatology" based on the data from the row-anomaly-free epoch between 2004 and $\sim$ mid-2008. At the last step we remove (subtract) these periodic patterns and bin the de-trended values into broad $30^{\circ}$ latitudinal zones. At every binning step the data are checked for $\pm 2 \sigma$ outliers. The procedure slightly differs for the UV1 and VIS radiances. The UV1 data are not partitioned into different reflectivity groups, while the VIS radiances are segregated into the low-, mid- and high-reflectivity (at $\lambda=388 \mathrm{~nm}$ ) categories at $r<10$ and $r>70 \%$ thresholds. To augment SNR (here the noise may be related to the inherently low signal level at the UV1 wavelengths; the "noise" could also be produced by the variable scenery in the VIS range), we use much broader, 2$3 \mathrm{~nm}$, wavelength bins in UV1 and VIS. We do not account for any SZA-related variability in the VIS data, delaying the removal of relevant trends until the climatology subtraction step (as above).

In Fig. 23 we plot the de-trended, binned UV1 and UV2 radiances for selected rows. The shown rows are very close to the main row-anomaly area (e.g., mainly image rows 25 42,54 and 55 in UV2, with occasional broadening of the row anomaly (RA)-affected area towards image rows 43-52). These "bordering" rows demonstrate relatively weak reaction to the on-going RA. Note the dominance of the blocking in the southern-hemisphere domain, latitude $-45^{\circ}$, and the interplay of the blocking (line-of-sight obscuration) and the solar stray light (December-January spikes at latitude $45^{\circ}$ ) in the northern-hemisphere radiances. The solar stray light is far more pronounced in the UV1 range, causing saturation of the UV1 detector at some rows, as well as affecting practically all UV1 rows at northern latitudes. For example, UV1 rows 12-22 (counting the row numbers from 1) are saturated at all wavelengths at high northern latitudes, lat $>=10^{\circ} \mathrm{N}$. The rows 23-27 show diminishing (with the increasing row number and increasing UV1 wavelengths) swathes of saturated radiances. Rows $1-11$ and $28-30$ can be considered as practically saturation free in the $\lambda>295 \mathrm{~nm}$ domain.

Figure 24 shows the de-trended row-, altitude- and wavelength-binned VIS radiances for the low-reflectance $(r<10 \%$ at $\lambda=388 \mathrm{~nm})$ sub-sample of the data. Besides the remarkably low instrument degradation, $\sim 1-1.5 \%$ between 2004 and 2015, one may notice $\sim 1-3 \%$ dip around 20092010. The feature becomes more pronounced as the row index moves closer to the RA-affected areas (rows $>23$ ). This 2009-2010 dip is seemingly absent in the high-reflectance category ( $r>70 \%$; not shown), with the latter being far less sensitive to the RA-related changes in the stray-light level. Hence, at some epochs (as shown for VIS) or at some orbital phases (as happens in UV1 $\lambda<300 \mathrm{~nm}$ radiances obtained at SZA $>45^{\circ}$ in the northern hemisphere) the RA-related changes may affect FOVs (rows) beyond the limits routinely flagged as RA-contaminated (as in Fig. 22). Various OMI L2 products show different sensitivity to the RA phenomena, 

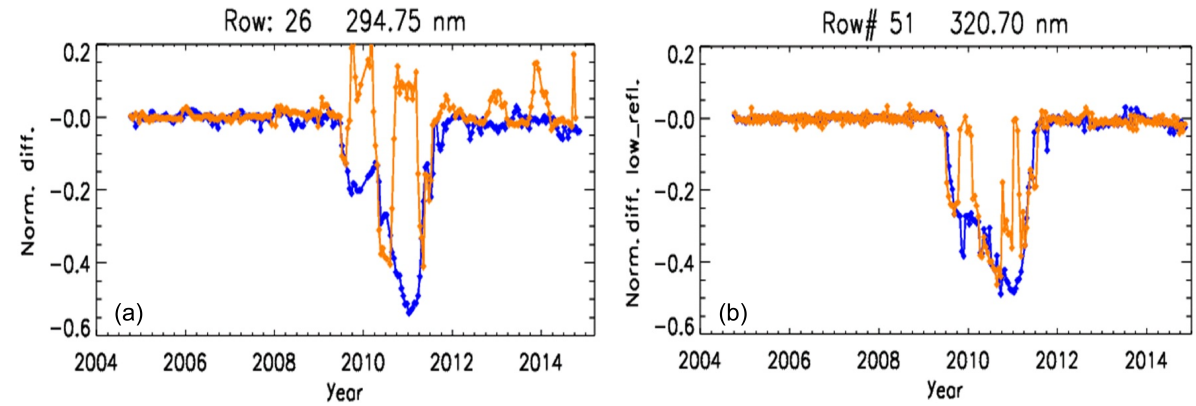

Figure 23. (a) Shows the wavelength-binned, normalized and de-trended UV1 radiances for the row 26 (counting from 1 ) for the $30^{\circ}$ latitudinal bins centered on latitude $-45^{\circ}$ (blue) and latitude $45^{\circ}$ (orange). (b) Shows the same for the UV2 low-reflectivity $(r<15 \%$ ) sub-sample of radiances registered by the row 51 (in correspondence to the FOV of the UV1 row 26). The plotting ranges correspond to $-60 \% /+20 \%$ changes of radiances.
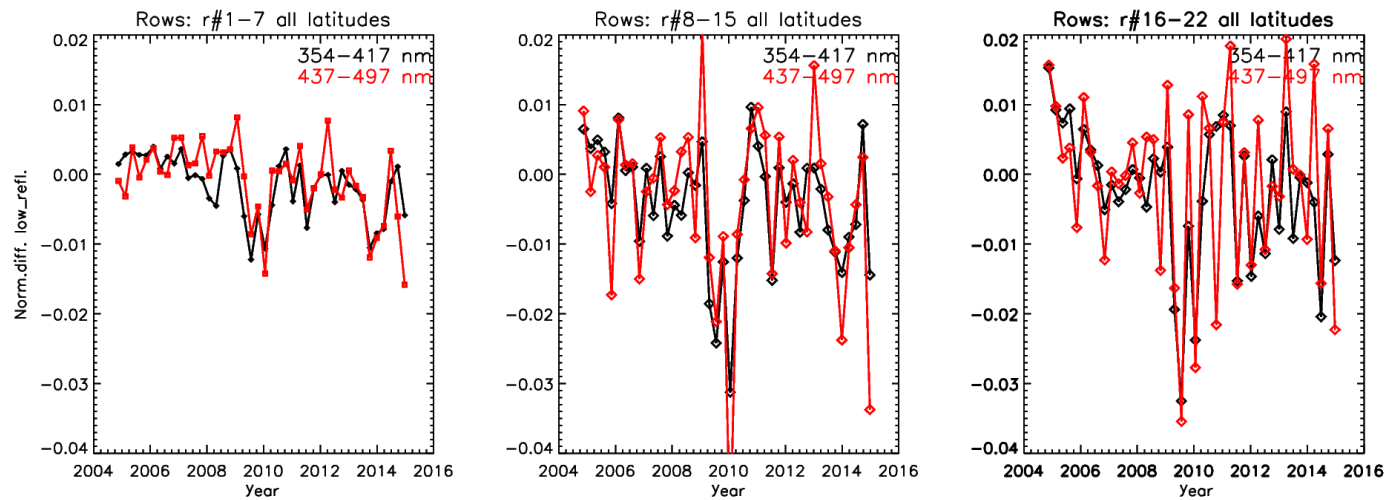

Figure 24. The time-, row- and wavelength-binned, normalized and seasonally de-trended VIS radiances sampled at all latitudes over the low-reflectance $(r<10 \%)$ terrain. All shown rows are presumably not affected by the row anomaly; though notice the changes around 2009-2010.

Table 4. Percentage of the RA-affected rows, as of August 2014.

\begin{tabular}{lrrr}
\hline Channel & UV1 & UV2 & VIS \\
\hline All orbit phases & $37 \%$ & $33 \%$ & $30 \%$ \\
Northern part of orbit phase & $100 \%$ & $57 \%$ & $52 \%$ \\
\hline
\end{tabular}

thus calling for a cautious interpretation of the OMI data in the bordering areas, e.g., UV2 rows $24,44-52$.

A single reliable method for the detection of the row anomaly has proven difficult to establish because the effects of the anomaly on radiances are complex and each science algorithm has its own sensitivities to radiance error that are difficult to capture with a single detection technique. The KNMI methods for detection through analysis of the OMI L1B radiances directly worked well to flag bad data from their L2 products but did not satisfactorily remove affected retrievals in some NASA L2 products. Therefore, an additional method was developed to determine the affected rows for the NASA algorithms, which is based on analysis of errors detected in the NASA TOMS L2 total ozone product. The NASA team developed its own row-anomaly detection scheme that identifies instrument error using a statistical analysis of total column ozone error. Total ozone anomalies are detected using data averaged in $5^{\circ}$ zonal mean bands by comparing the rowto-row behavior of the data to a baseline OMI dataset similarly constructed from data collected prior to the onset of the row anomaly (Haffner, 2012). Total ozone is a good basis for the anomaly detection because algorithm errors are relatively well understood and the mean geophysical behavior of total ozone can be thoroughly characterized. Though the NASA flags were originally designed for total ozone, they also work well for other products such as $\mathrm{SO}_{2}$, which is very sensitive to radiance errors, and also the OMI aerosol optical depth and single scattering albedo product derived from the VIS detector. A comparison between the results from the NASA and KNMI flagging results is shown in Fig. 25. In this figure, no distinction is made between the four different row-anomaly effects or if a row is flagged for the entire orbit or just a part of the orbit. This figure nicely summarizes the percentage of OMI detector rows that are affected by the anomaly as a function of time, as detected in the KNMI L1B monitoring approach and the NASA method. Both indicators 


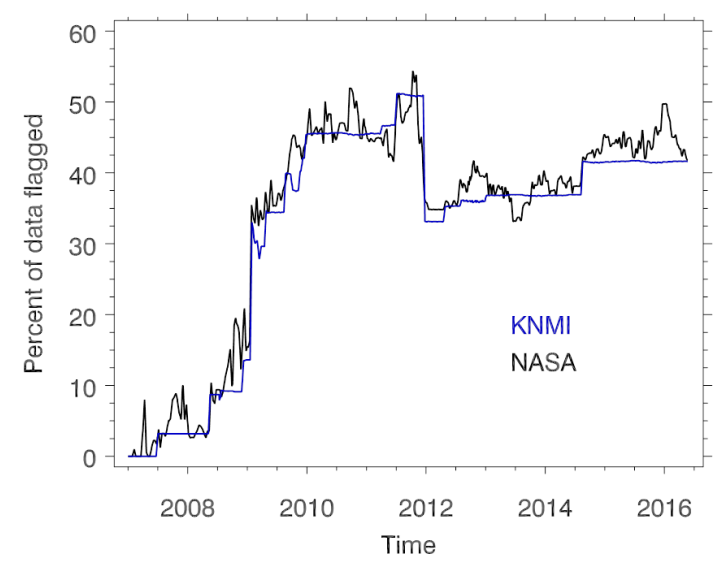

Figure 25. Percent of data flagged by the two row-anomaly flagging algorithms in use for the UV2 channel. The KNMI algorithm is used to flag the L1B radiance products, while the NASA algorithm is used in several NASA retrieval algorithms to flag the L2 data. Though the physical basis of the two algorithms is rather different, they produce consistent flagging results over the full course of the OMI mission. The presence of high-frequency variations in the NASA flagging algorithm is due to the fact that it flags data dynamically, while the KNMI row-anomaly flags are changed as need determines.

track the anomaly similarly, but differences do exist in how the flags are set for some data.

\section{Long-term calibration}

\subsection{Status of current Collection $3 \mathrm{~L} 1 \mathrm{~B}$ products}

The L1B products that are produced are part of the Collection 3 data. Collection 3 data was started on 1 February 2010 with the introduction of version 1.1.3 of the L1B processing software (Ground Data Processing System, GDPS; Dobber et al., 2008a). When version 1.1.3 was introduced, all data since the beginning of the OMI mission has been reprocessed with this version. The main improvements in version 3 are a more elaborate flagging of the row-anomaly effects, new wavelength fit coefficients, improved stray-light correction in UV2, and an improved noise calculation. A one-time adjustment to the radiometric calibration was also applied. There were no changes in the basic flow of corrections on the data products. These corrections are shown in Table 4. More extensive information with, for example, flagging functions can be found in Oord et al. (2006). The generic functions in the table are executed for all measurements. Depending on the measurement type an extra series of correction functions is applied. There has been an effort to make correction functions for the row-anomaly effects, but these corrections did not give the desired results. It is difficult to separate the different row-anomaly effects, and therefore they could not be

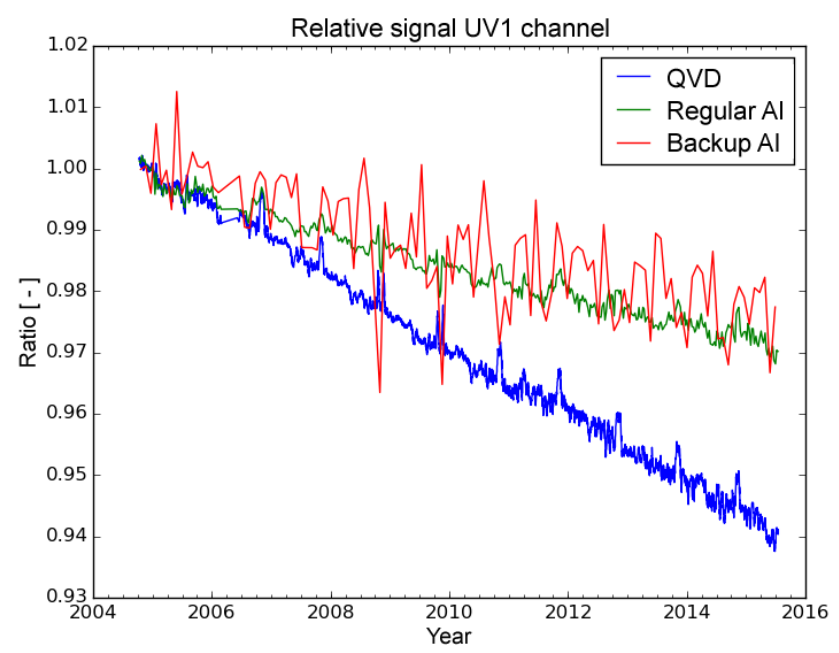

Figure 26. Relative solar signal of the UV1 channel over the mission. Each data point shown for the three diffusers is a result of a spectral and spatial average over the entire channel. The higher rate of signal change from the frequently used QVD suggests degradation in diffuser reflectivity caused by solar exposure.

corrected satisfactorily. There are only flagging functions for the row-anomaly effects.

\subsection{Radiometric calibration}

This section addresses changes in instrument radiometric calibration as observed in the solar measurements and the Earth radiance measurements. Each observational port may provide an independent view of sensor changes since launch that may not be necessarily consistent. This is particularly true for OMI, where the optical paths differ for Earth-view and solar measurements. The challenge is to reconcile these differences and to describe as accurately as possible the calibration changes in the Earth radiance path. We begin with a discussion of the solar measurements.

Solar calibration measurements are performed every day. In a solar calibration measurement the sunlight passes via the mesh through the opened solar aperture onto a reflective diffuser: either the aluminized fused silica (QVD hereafter), or two pure Aluminum diffusers. The reflected sunlight is coupled into the instrument telescope via the folding mirror (see Fig. 5).

The relative solar signal in the UV1 channel for the three diffusers is shown in Fig. 26. In this figure a solar measurement is divided by a reference solar measurement from the beginning of the mission. The average of this ratio for the UV1 channel is calculated and shown in the figure. If we assume the three react similarly to solar exposure, their differences appear to be related to their frequency of exposure. The QVD is used every day, the regular Al diffuser once per week, and the backup Al diffuser once per month. This leads to less degradation of the aluminum diffusers. The changes 
Table 5. Correction functions for the different measurement types. Generic corrections are applied to all measurement types.

\begin{tabular}{|c|c|c|c|c|c|c|c|c|c|c|c|c|}
\hline Measurement type & \multicolumn{12}{|c|}{ Correction functions } \\
\hline \multirow[t]{2}{*}{ Generic } & \multicolumn{12}{|c|}{$\begin{array}{l}\text { Co-addition division, ADC conversion, offset correction, } \\
\text { gain overshoot correction, electronic conversion, non-linearity correction, } \\
\text { binning factor division, offset calculation, and calculation of measurement noise }\end{array}$} \\
\hline & 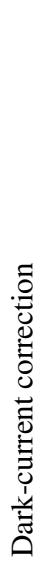 & 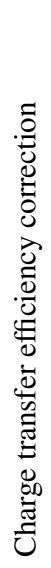 & 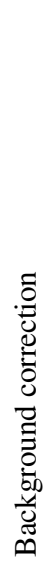 & 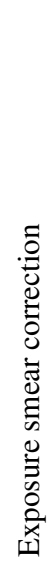 & 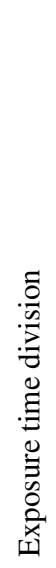 & 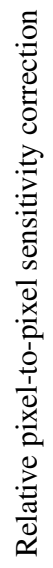 & 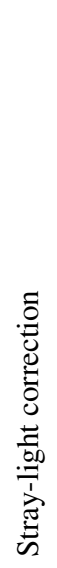 & 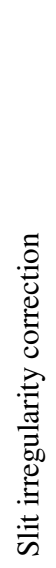 & 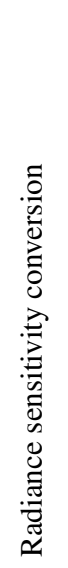 & 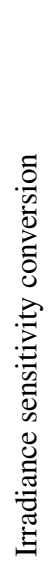 & 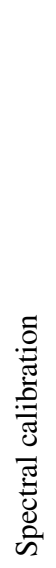 & 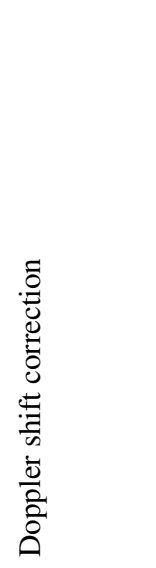 \\
\hline Earth & $\mathrm{x}$ & $\mathrm{x}$ & $\mathrm{x}$ & $\mathrm{x}$ & $\mathrm{x}$ & $\mathrm{x}$ & $\mathrm{x}$ & $\mathrm{x}$ & $\mathrm{x}$ & & $\mathrm{x}$ & \\
\hline Sun & $\mathrm{x}$ & $\mathrm{x}$ & $\mathrm{x}$ & $\mathrm{x}$ & $\mathrm{x}$ & $\mathrm{x}$ & $\mathrm{x}$ & $\mathrm{x}$ & & $\mathrm{x}$ & $\mathrm{x}$ & $\mathrm{x}$ \\
\hline WLS & $\mathrm{x}$ & $\mathrm{x}$ & $\mathrm{x}$ & $\mathrm{x}$ & $\mathrm{x}$ & & $\mathrm{x}$ & $\mathrm{x}$ & & & & \\
\hline LED & $\mathrm{x}$ & $\mathrm{x}$ & $\mathrm{x}$ & $\mathrm{x}$ & $\mathrm{x}$ & & & & & & & \\
\hline Dark & & $\mathrm{x}$ & & & & & & & & & & \\
\hline
\end{tabular}
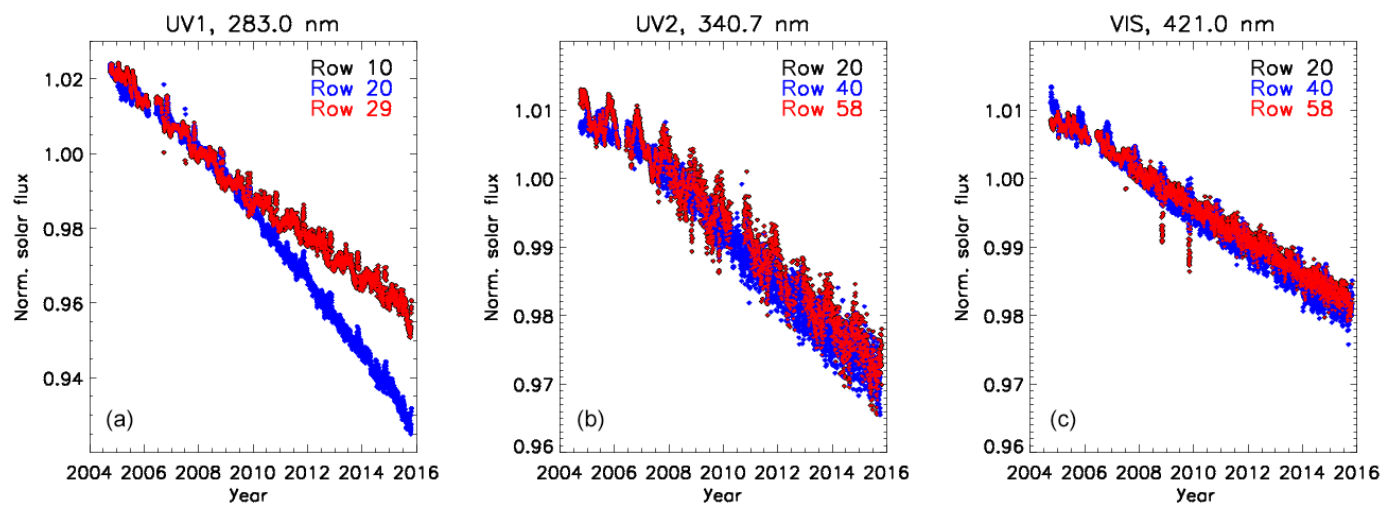

Figure 27. The wavelength-binned and normalized QVD solar irradiances for different rows in the UV1 (a), UV2 (b) and VIS (c) channels. The increase in change with decreasing wavelength is typical of optical degradation related to solar exposure. The anomalous change in the UV1 row 20 beginning in 2009 may be caused by additional solar exposure resulting from the row-anomaly reflections.

observed in all channels are provided in Table 5. For the UV1 channel, the signal change is $6 \%$ for QVD, $3 \%$ for regular $\mathrm{Al}$ and $2.5 \%$ for backup $\mathrm{Al}$. These are overall signal changes of the complete instrument. Since the backup diffuser is used so little, the signal change of $2.5 \%$ can be attributed to the complete instrument.

To further substantiate our hypothesis of QVD optical degradation, we zoom in into individual wavelength bands in Fig. 27. The bands with the shortest wavelengths have the largest degradation. An exception to this is the $372-376 \mathrm{~nm}$ band in UV2 (not shown), which tends to degrade slightly (by $\sim 0.5 \%$ over 10 years) faster than expected for the particular wavelength range.

We attribute the accelerated degradation of row 20 in UV1 starting in 2009 to scattered sunlight during northern hemisphere Earth-view measurements. This is described as the solar contamination effect in Sect. 5. Assuming this is the cause, the change is likely occurring in the telescope assembly. This follows because the diffusers, as well as the folding mirror, are not in the optical path during Earth-view mea- 


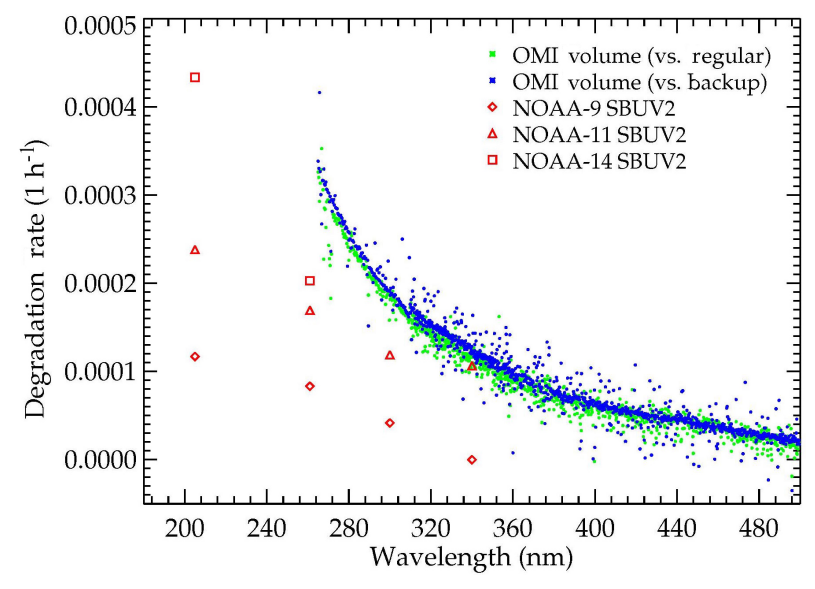

Figure 28. The fractional change in the QVD diffuser per hour of solar exposure relative to the regular and backup Al diffuser.
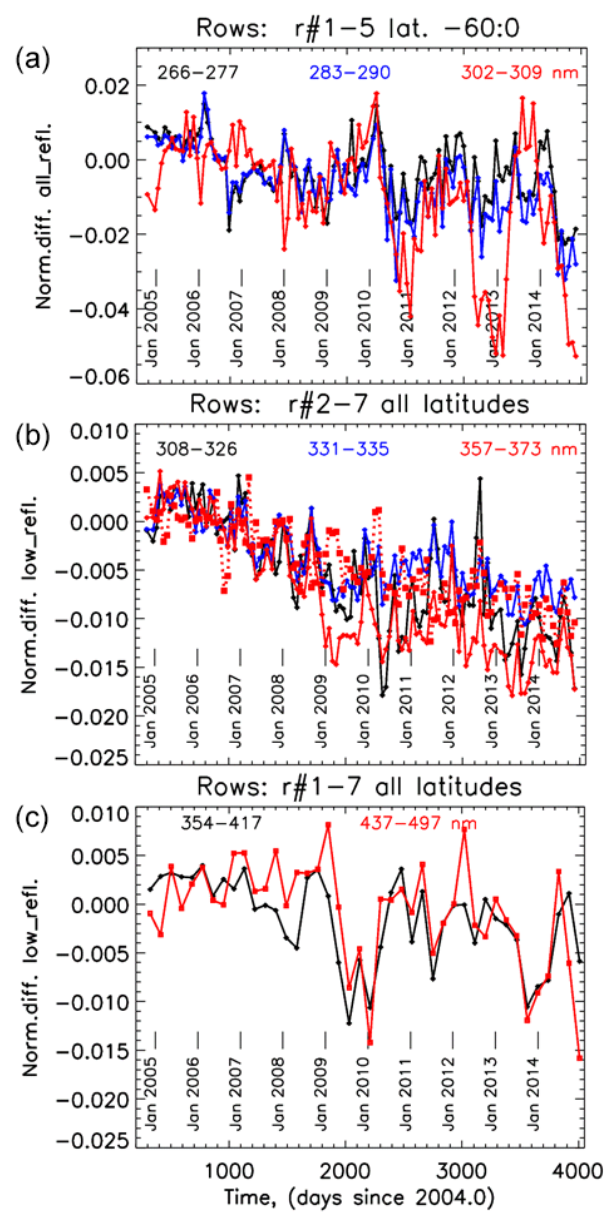

Figure 29. (a) The de-trended, normalized time-, wavelength-, latitude- and row-binned UV1 radiances. (b) The same for UV2 for the low-reflectivity $(<10 \%)$ subsample (full red line) and the high-reflectivity (>80\%) data (dotted red line) for the $357-373 \mathrm{~nm}$ range. (c) The low-reflectivity, binned radiance trends for VIS. Note the change of the $y$-axis plotting scales for different OMI channels.

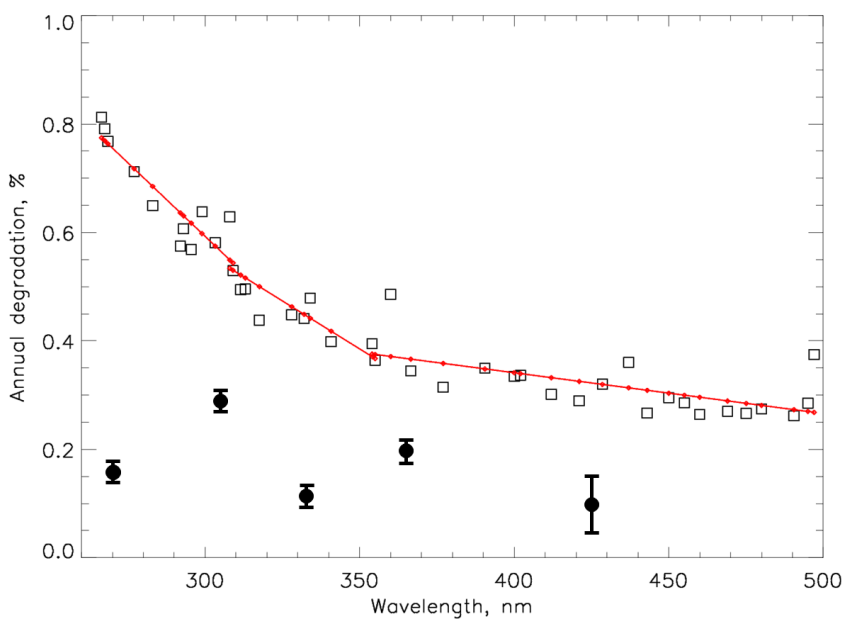

Figure 30. The annual signal change rates (QVD diffusor, squares) derived from the wavelength-binned solar irradiances for 20072009 (see more details in Marchenko and Deland, 2014) and fitted with linear polynomials (red lines) for each OMI channel. Filled circles and $\pm \sigma$ error bars show the degradation estimates in the OMI radiances.

surements (Fig. 5); hence, they are not exposed to the anomalously scattered solar light. The primary telescope mirror is bypassed for the solar measurements, so the row 20 anomaly in Fig. 27 is likely caused by accelerated degradation of the secondary telescope mirror. We surmise that the degradation of the primary mirror is even greater for the RA-affected across-track positions since it is the first optical element exposed to the RA-scattered solar light. The interference with other row-anomaly effects makes it impossible to verify this hypothesis.

We can isolate the optical degradation of the solar diffusers by comparing the signal changes observed with each one. Figure 28 shows the fractional change in the QVD per hour of solar exposure relative to the other two diffusers. The close agreement between QVD changes derived from the regular and backup diffusers is an indication that neither has degraded significantly. If we assume the regular and backup diffusers have similar degradation rates, the former should be degraded more than the latter in a ratio of $32 / 7$. A substantial degradation of either would result in a separation of the blue and green points in Fig. 28.

Closer examination of regular diffuser change relative to the backup reveals a rate of $1.10^{-4} \mathrm{~h}^{-1}$ at $265 \mathrm{~nm}$, which is significantly less than the $3.10^{-4} \mathrm{~h}^{-1}$ observed for volume diffuser change. This difference is important because the multi-diffuser approach to calibration relies on equal degradation rates. For the results presented here we have assumed that the backup diffuser has not degraded, but in fact a small correction is required for its change based on the regular diffuser degradation rate. Since that rate is already very low it is unlikely that an error in the backup degradation rate, even if it were half that of the regular's, would result in a signif- 
icant calibration error. Still, these results should serve as a warning to minimize the exposure of the least-used diffuser for fear that uncertainties in its degradation rate may become a significant component in the calibration error budget.

The observed QVD degradation rates are similar to those seen for several of the SBUV2 instruments, though the OMI QVD appears to have a steeper wavelength dependence. It is noteworthy that the OMI solar measurements employ a protective mesh in front of the diffuser that attenuates the incident solar irradiance by approximately a factor of 10 . This implies that the QVD degradation rate per equivalent solar exposure is much larger than that of the SBUV2 diffusers and larger even than that of the TOMS diffusers (see Jaross et al., 1998). The design and operational factors affecting degradation rates are complex and to this day not fully understood. This underscores the importance of maintaining low exposure frequencies until on-orbit rates become clear.

The degradation of the OMI instrument downstream of the diffusers is approximated by the change observed in the backup measurements (see Table 5) because the expected degradation of that diffuser is so small. To assess how these non-diffuser changes affect the OMI Earth-view measurements requires an independent estimate of measured radiance change. The estimated changes, shown in Fig. 29, are assessed by removing common seasonal and other cyclic variations. No attempt has been made to remove any putative long-term geophysical changes from the radiances.

Instrument changes related to Earth-view measurements are summarized in Fig. 30. Signal changes for the solar QVD measurements, shown in the same plot, are significantly greater. Note that in the UV region $(\lambda<300 \mathrm{~nm})$ natural variations can exceed $1 \%$ at typical solar cycle time scales (> 5 years: Marchenko and Deland, 2014). The shown QVD degradation changes are corrected for this variability. Falling substantially below the QVD values, the Earth radiance rates are nevertheless consistent with solar signal changes measured with the backup diffuser (cf. Table 5). This suggests that for rows not affected by the row anomaly, the change in the primary telescope mirror is negligible, and the change in radiometric calibration can be accurately estimated using the backup diffuser solar measurements, once the solar variability factors are taken into consideration.

The derived Earth radiance changes are confirmed by observing measured signals (Fig. 31) over Greenland and Antarctica. Assuming that the mean reflectivity of the ice surfaces has not changed over the OMI mission, we conclude that the optics and detector have changed by $\sim 1-1.5 \%$ at $360 \mathrm{~nm}$ in UV2 for rows far from the row-anomaly blockage. Substantial changes are observed near the RA-affected nadir view (right panel in Fig. 31), where the blockage is the greatest.

This conclusion is substantiated by the long-term trends seen in the OMI radiances (Fig. 29). In this figure we show de-trended and wavelength-, latitude-, time- and row-binned radiances. As an example, for UV1 we select all the available data for image rows $6-10$, which are practically unaffected by RA at southern-hemisphere latitudes. We show image row 2-7 UV2 trends for all latitudes and the radiances from the low-reflectivity $(<10 \%)$ subsample of the data. In addition, we show the trend for the high-reflectivity $(>80 \%)$ group for the 357-373 nm wavelength bin, keeping in mind that the onset of a major RA event in January 2009 may have changed the OMI stray-light levels due to the physical blocking of some Earth-viewing angles as well as additional scattering of the Sun and Earth light. Hence, if the currently implemented stray-light correction does not adequately capture the RArelated changes, one should see different temporal behavior among the high- and low-reflectivity sub-samples. The lowreflectivity scenery turns out to be $\sim 5$ times more susceptible to the stray-light contamination than the high-reflectivity data. Indeed, we see close agreement between the highreflectivity (dotted red line in Fig. 29) and low-reflectivity (full red line) UV2 trends, which abruptly changes at the beginning of 2009. However, the overall effect is rather small, reaching $\sim 1 \%$ for the long-wavelength UV2 range and consistently staying below $1 \%$ (our sensitivity limit in detecting the long-term trends in radiances) in the $310-350 \mathrm{~nm}$ UV2 range. Chromatic terms in the radiance trends are relatively small for UV1 and UV2 $(<\sim 2$ and $<\sim 1 \%$ over the mission time, respectively) and practically absent $(<0.5 \%$, our sensitivity limit in detecting the chromatic trends) in VIS.

We directly examined the ratios of Sun-normalized radiances measured at wavelengths separated by several nanometers to confirm that there is little change in the spectral dependence of OMI's overall radiance calibration over the course of the mission. The data shown in Fig. 32, for three of the OMI detector rows unaffected by the row anomaly, are selected to minimize natural sources of trend and variability in the ratios of radiances. The main geophysical effects which introduce time-varying spectral dependence in backscattered Earth radiances are Rayleigh and aerosol scattering, trace-gas absorption, and Raman scattering. Because these effects are much larger than the changes in the instrument spectral dependence, and they are highly variable over space and time, particularly over shorter scales, it is useful to isolate certain data so the impact of these effects on the radiances is reduced. A total of $354 \mathrm{~nm}$ radiances are unaffected by trace-gas absorption, and while 340 and $380 \mathrm{~nm}$ radiances have minor $\mathrm{O}_{3}$ and $\mathrm{O}_{2}-\mathrm{O}_{2}$ absorption, these effects are small and we limit their impacts on radiance trends by restricting our data to the tropics where the variability in total ozone is small. We select data measured over the central Pacific in the tropics, a region removed from sources of dust and smoke that would contaminate the spectral ratios. To reduce the effect of Rayleigh and Raman scattering we restrict ourselves to data measured over very bright convective clouds, which we select by requiring that the top of the atmosphere (TOA) reflectance, $\rho$ at $340 \mathrm{~nm}$, is greater than 0.9 , and we calculate $\rho=\pi^{*} I / F^{*} \sec \left(\theta_{0}\right)$, where $\theta_{0}$ is the solar zenith angle and radiance $I$ is normalized by solar flux $F$. The large values of 

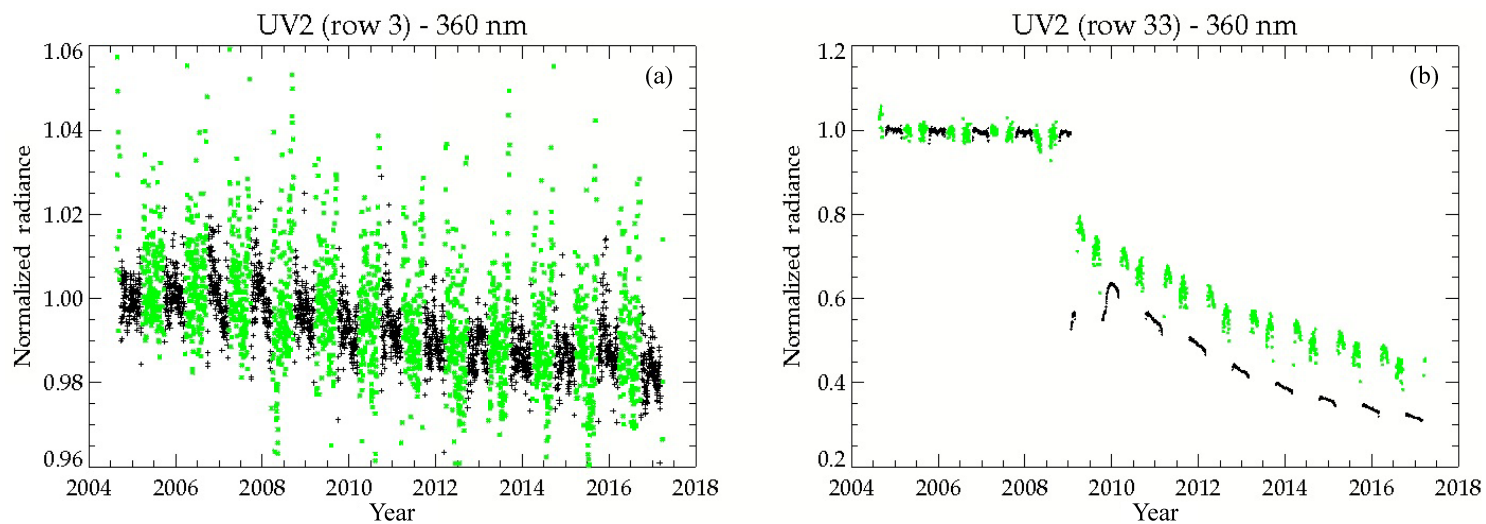

Figure 31. Measured daily mean radiances in UV2 at $360 \mathrm{~nm}$ over Antarctica (black) and Greenland (green) are shown as a function of time relative to initial measurements in 2004. Left panel shows results in row 3, which is at the western edge of the OMI swath and is far from the row-anomaly-related blockage. Right panel shows results for row 33, which is near nadir and is affected by RA.
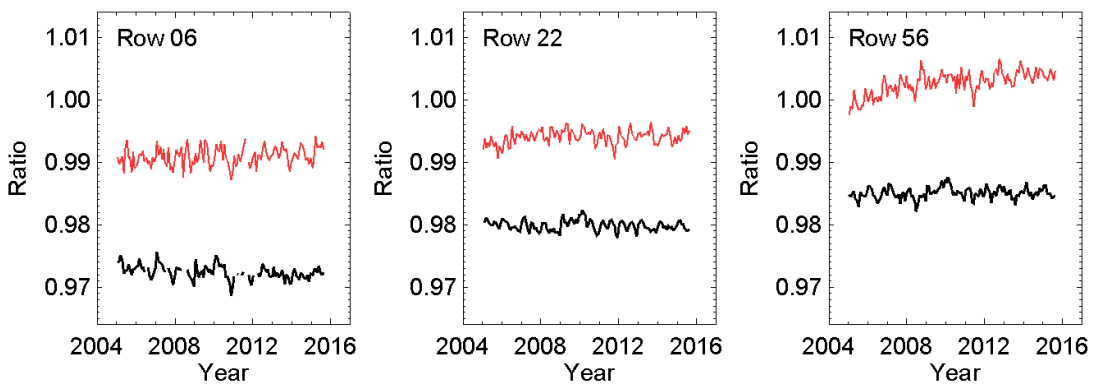

Figure 32. Trends in monthly mean ratios of OMI radiances measured at 340 and $354 \mathrm{~nm}$ on the UV-2 detector (black) and 354 and $380 \mathrm{~nm}$ on the VIS detector (red) for three detector rows. The observed trend in these Earth-view measurement ratios is $<0.5 \%$ per decade in both channels. Detector rows 6, 22, and 56 shown here are among those which are unaffected by the row anomaly. The data shown here were selected to minimize geophysical effects of wavelength dependence and measurement geometry on the radiance ratios. Conditions are limited to high reflectance $(\rho>0.9)$, low solar zenith angle $\left(\theta_{0}<50^{\circ}\right)$ over the tropical Pacific $\left(20^{\circ} \mathrm{N}-\mathrm{S}, 130-170^{\circ} \mathrm{W}\right)$, where radiation is scattered primarily by high altitude, and deep convective clouds and the observed atmosphere is generally free of aerosol. The more noticeable trend in row 56 is not understood. Despite specifically selecting data to minimize wavelength dependent effects, some residual geophysical variation remains in the ratios. These are most likely due to cloud scattering phase function variation with cloud water phase and from residual multiple scattering effects varying seasonally over the SZA range of the measurements.

TOA reflectance over the tropical oceans are associated with mature deep convective clouds whose cloud top pressures are near $200 \mathrm{hPa}(\sim 12 \mathrm{~km})$ and optical centroid (effective) cloud pressures inside the cloud are near $500 \mathrm{hPa}(5.6 \mathrm{~km})$ because of appreciable penetration into the cloud of photons subsequently scattered back to the top of the atmosphere (Vasilkov et al., 2008; Ahmad et al., 2004). These clouds are of such high reflectance and at altitudes well above a significant portion of the atmosphere that the ratio of direct to diffuse scattered radiation received by OMI is significantly enhanced by the presence of these clouds, which reduces the variation in Rayleigh scattering due to changes in solar zenith angle with season. The largest values of solar zenith angle for the data considered here are $50^{\circ}$.

The results in Fig. 32 show the monthly means of the wavelength ratios of 340 to $354 \mathrm{~nm}$ on the UV-2 detector and 354 to $380 \mathrm{~nm}$ from the VIS detector, for three detector rows over the course of the OMI mission (useful $354 \mathrm{~nm}$ measurements are made on both the UV-2 and VIS detectors). The trends in these ratios are less than $0.5 \%$ per decade.

Small seasonal and interannual variations remain in the radiance ratios despite our efforts to minimize the wavelength dependent geophysical effects on the variability of these data. These variations are most likely from remaining geophysical effects such as residual solar zenith angle dependence, variation in cloudiness, and possibly aerosol contamination caused by volcanic events. They are not thought to be related to detector performance. The somewhat greater amplitude of the variations seen for the VIS detector ratios can be explained by the fact that the leading spectrally dependent effects at these wavelengths increase with greater wavelength separation, and the wavelengths on VIS are separated by $26 \mathrm{~nm}$, whereas those on UV-2 are separated by $14 \mathrm{~nm}$. This analysis is limited to the UV-2 and VIS detectors be- 


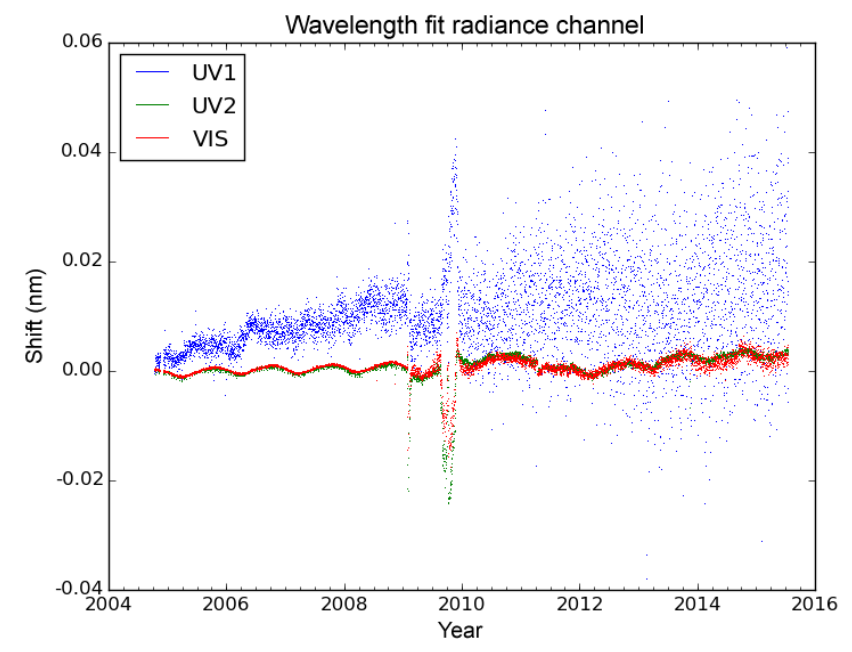

Figure 33. Spectral calibration trend over the mission for the radiance channel. Spectral calibration for the UV1 channel is strongly affected by the row-anomaly effect since 2009 .

Table 6. Solar signal changes observed in the three channels for the timeframe 2005-2015.

\begin{tabular}{lrrr}
\hline & UV1 & UV2 & VIS \\
\hline QVD & $6 \%$ & $4 \%$ & $3 \%$ \\
Regular Al & $3 \%$ & $2 \%$ & $2 \%$ \\
Backup Al & $2.5 \%$ & $2 \%$ & $2 \%$ \\
\hline
\end{tabular}

cause radiances measured by UV-1 are much more sensitive to ozone, and the ozone absorption cross section varies dramatically over just a few nanometers in that detector's spectral range.

\subsection{Spectral calibration}

Level 2 retrievals which use differential optical absorption spectroscopy or principal components analysis techniques to derive trace-gas information from the high spectral frequency structure in Level 1 reflectance measurements can be sensitive to wavelength errors as small as 1/100th of the OMI wavelength sampling interval (van Geffen et al., 2015; Marchenko et al., 2015). During the mission spectral calibration is performed as described in Sect. 3.2 and Appendix A. The in-flight spectral calibration coefficients are calculated using two different methods. In the first method the preflight calibration parameters are modified by a function using the optical bench temperature and a correction for inhomogeneous slit illumination. For the second method the calibration coefficients are determined using a fit of the measured spectrum with a high-resolution solar spectrum, augmented with an ozone spectrum and a Ring spectrum.

The changes of calibration parameters based on the fit results (the second wavelength calibration method) are shown in Fig. 33. Here we plot the first polynomial coefficient $\left(c_{\text {fit }, 0}(j)\right.$ in Eq. A16) for image row $j=15$ for UV1, and image row $j=30$ for UV2 and VIS. Note that the radiance measurements at these rows are affected by the row anomaly, which greatly exacerbates the scatter in the UV1 values after the major January 2009 RA event. This particular polynomial coefficient (in the plots we show the daily averages) is used to demonstrate the long-term stability of the instrument. The wavelength registration gradually drifts by $0.015 \mathrm{~nm}$ in UV1, and for the UV2 and VIS channel it shows remarkable stability, changing by $\sim 0.002 \mathrm{~nm}(\sim 1 / 100$ of a pixel) over the 10-year span. We also see that the trend in the shift of the wavelength registration follows that of the temperature of the optical bench (cf. Fig. 21). Therefore, the initial expectation that the wavelength registration depends mainly on the temperature of the optical bench, seems to be fulfilled.

We complement the findings from Fig. 33 by trending the wavelength registration provided via the first calibration method, i.e., the preflight calibration adjusted by the OPB temperature and, whenever applicable, the inhomogeneous slit illumination. As an example, we take the daily OMI irradiance measurements, and for each row we select prominent spectral lines spanning the sensitivity ranges of the OMI spectral channels. For each row in each channel we calculate centroids of these prominent absorptions. For a given line, in all rows the calculated centroids show similar, to within sensitivity limits, time dependencies. Hence, we average all the UV2 and VIS rows, however, limiting the averages to the UV1 image rows 1-13, thus avoiding the FOVs experiencing anomalous degradation rates (see Fig. 27). We additionally average values over 3 consecutive months and subtract the early-mission estimates from the line-centroid measurements. Figure 34 shows changes in the line centroids for the selected representative lines. Over the mission time, line positions gradually shift at $<\sim 0.005 \mathrm{~nm}$ pace in UV1, save the $\sim 290-300 \mathrm{~nm}$ region where the drift practically triples. The UV2 and VIS channels show progressively lower changes, with long-term trends in the latter not exceeding the scatter levels, $\pm 0.001 \mathrm{~nm}$, probably related to the yearly oscillations of the OPB temperature.

The long-term (mission time) and short-term (orbital) stability of the instrument spectral response function is deemed important for reliable, unbiased retrievals of the atmospheric trace-gas properties. Changes in the instrument spectral response affect depths and widths of the detected spectral features. In Fig. 35 we show variations of the line-profile parameters derived from radiances for the line blend around $\lambda=336.1 \mathrm{~nm}$ and the UV2 row 5 . Each panel shows the differences between the latitude- and time-binned early-orbit (latitude is -60 to $-50^{\circ}$ ) and late-orbit (latitude is 40 to $50^{\circ}$ ) line-profile parameters. The orbit-differentiated wavelength registration and FWHMs go through relatively minor $( \pm 0.001 \mathrm{~nm})$ seasonal changes which we deem negligible in comparison to the $0.14 \mathrm{~nm}$ UV2 sampling rate. The linedepth variability show clear $\pm 0.2 \%$ seasonal fluctuations, most likely related to changes in the Ring-line-filling factors, 

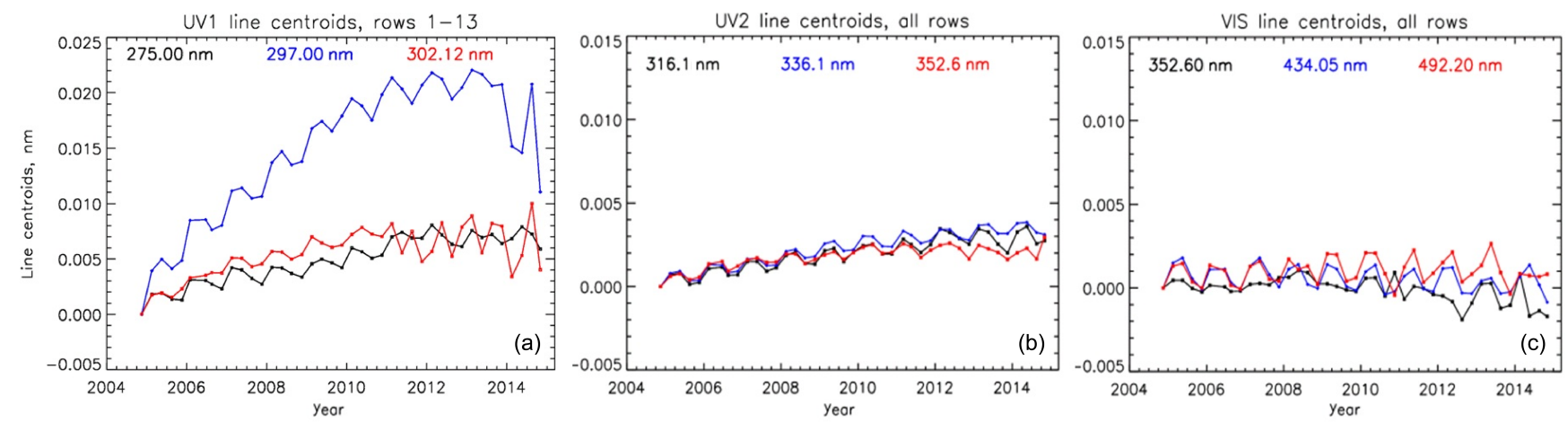

Figure 34. Changes in the line centroids of prominent absorption blends in the solar irradiances observed in UV1 (a), UV2 (b) and VIS (c).
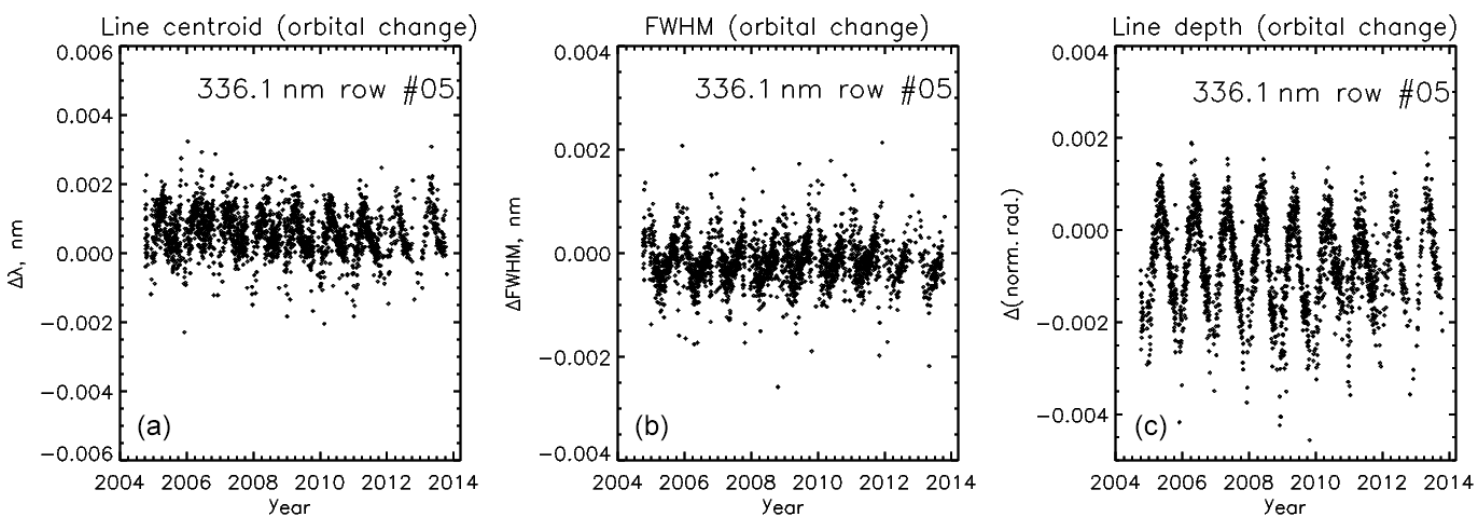

Figure 35. The orbital changes of the line centroids (a), line FWHMs (b) and line depths (c) in the UV2 radiances for the spectral blend at $336.1 \mathrm{~nm}$ and the row 5 . These trends are representative for all rows.

with their direct proportionality to the seasonably changing (solar elevation for a given latitude) atmospheric pathlengths. The line centroids are also involved in $\pm 0.001 \mathrm{~nm}$ seasonal cycling. However, such fluctuations should be regarded as negligible in comparison to the $0.142 \mathrm{~nm}$ sampling rate in the UV2 spectra. The shown trends are representative for all rows. Hence, we may conclude that instrumental factors do not introduce observable (i.e., exceeding our sensitivity limits) spectral response changes along the OMI orbit nor do such factors cause any long-term (mission time) instrumental trends exceeding $\sim 0.2 \%$ in measurements of the UV2 and VIS absorption features (see Fig. 20).

Many trace-gas retrieval algorithms rely on solar reference spectra, thus creating an additional dilemma: should the referencing use static (usually, chosen at the beginning of the mission) or dynamic solar data? Choosing between two approaches, one should take into consideration a multitude of conflicting requirements. Among them is the possible different degradation rates in the optical channels acquiring radiance and irradiance data. OMI shows such differences, though they are relatively small. Also, one has to consider the gradual SNR decrease in the solar data (cf. Fig. 18). Even in the moderate-resolution OMI irradiances, the daily, monthly and long-term solar variability is prominent in the lambda $<450 \mathrm{~nm}$ domain, frequently exceeding $0.5 \%$ in the strong spectral blends (Marchenko and DeLand, 2014), calling for a thorough evaluation of sensitivity of the L2 science products to the variable solar spectrum. On the other hand, any substantial (far exceeding the sensitivity of a typical tracegas retrieval algorithm) long-term wavelength drifts may require extensive interpolations of the static solar data, thus augmenting the under-sampling biases (Kurosu et al., 2004).

The frequently used in the OMI L2 applications static solar spectrum was produced (T. Kelly, personal communication, 2017) from seven subsequent daily solar observations acquired between 28 December 2004 and 3 January 2005. This static reference spectrum is derived as an unweighted average of the daily observations, neglecting exceedingly large deviations from the corresponding row (i.e., FOV) and wavelength-dependent median values.

\section{Conclusions, summary and outlook}

Analyzing long-term trends in the OMI L1B products, as well as values of calibration parameters, we conclude that, apart from the ongoing row anomaly, the instrument contin- 
Table 7. OMI performance over the mission time, year 2005-2015.

\begin{tabular}{ll}
\hline Characteristic & Change \\
\hline Dark current & $7 \times$ increase \\
RTS warnings & $0.7 \%$ increase \\
Bad pixel warning & $1.4 \%$ increase \\
Monitoring source LED & $85 \%$ of the original value \\
Monitoring source WLS & $90 \%$ of the original value \\
Gain ratios & $\sim 0.2 \%$ fluctuations \\
Electronic offsets & $\sim 0.5 \%$ fluctuations \\
Non-linearity warning & No significant changes since launch \\
Stray-light warning & No significant changes since launch \\
Irradiance decrease & $\sim 6 \%$ QVD diffuser; $3 \%$ regular Al; $2.5 \%$ backup Al \\
Radiance decrease & $\sim 2.5 \%$ UV1; $\sim 1.5 \%$ UV2; $\sim 1 \%$ VIS \\
Wavelength shifts & $\sim 0.015$ nm in UV1; 0.005 nm in UV2 and VIS \\
Current status of the row anomaly: & UV2 and VIS: $>\sim 30 \%$ and up to $\sim 57 \%$ (part of the northern hemisphere) \\
$\%$ of the affected rows & (southern hemisphere) \\
& UV1: $>23 \%$ (southern hemisphere) and all rows \\
& (part of the northern hemisphere) \\
\hline
\end{tabular}

ues to perform well. Though still gradually unraveling, the row anomaly remains relatively stable since the latest incident in the summer-fall 2011.

One of the most noticeable trends is the 7-fold darkcurrent increase. The RTS warnings, which are closely related to the dark-current readings, have increased to $0.7 \%$ of the CCD pixels used for data acquisition, or to $5.6 \%$ if one considers the binning factors ( $8 \mathrm{CCD}$ pixels $=1$ row) used in the L1 and L2 OMI products. Bad-pixel warning has increased to $1.4 \%(11.2 \%)$. The output of the monitoring lamps, LED and WLS, decreased to 85 and $90 \%$ of the earlymission values, respectively. However, this has not hampered the routine calibration activities. There are some fluctuations in the gain ratios and electronic offsets, but they are small and do not impact the signal processing routines. The nonlinearity warnings and stray-light warnings remain stable.

The gradual decrease in the irradiance values can be primarily attributed to degradation in the solar diffusers. The daily measured irradiances from the QVD diffuser decreased by $6 \%$ in the UV1 channel. The output from the Al diffuser, which is used once per week, diminished by $3 \%$ in the same wavelength range. The rarely (once per month) used backup Al diffuser has degraded by $2.5 \%$ in the UV1 channel. Since the exposure times for the weekly and monthly calibration cycles differ by 4 times, it can be safely assumed that the registered $2.5 \%$ degradation of the backup $\mathrm{Al}$ diffuser is attributable to the complete optical pathway of the OMI irradiance measurements (Fig. 2). We also register the substantially ( $\sim$ twice) higher degradation pace in the UV1 rows affected by the row anomaly. The longer-wavelength OMI channels show progressively lower degradation, with daily QVD values dropping by $\sim 3 \%$ over the mission time.

In the RA-free areas the trends in the OMI radiances point to surprisingly small changes, with good consistency among the UV1, UV2 and VIS channels: the UV region shows a $\sim 2 \%$ downward trend, while at visible wavelength the longterm changes amount to $1-1.5 \%$ over the mission time.

The long-term wavelength drifts in the UV2 and VIS channels do not exceed $0.005 \mathrm{~nm}$, attesting to excellent thermal and mechanical stability of the instrument. Gradual drifts in the UV1 range amount to $0.015 \mathrm{~nm}$, with some evidence of wavelength dependence.

We perceive the row anomaly as the most formidable instrument problem that renders unusable a significant proportion of the RA-affected rows (FOVs). The anomaly was unequivocally detected in two rows in June 2007. Alternative approaches point to the possibility of an even earlier incursion around the fall 2005-winter 2006. In May 2008 a large new group of rows became affected. The row anomaly continued to develop since then, with the particularly swift changes around January 2009 and the early fall of 2011. It has been relatively stable since then. The latest small increase in the affected rows dates to August 2014. Overall, the numbers of the RA-affected rows depend on the OMI channel, with radically different latitudinal and seasonal behavior in the UV1 and UV2 channels and comparable patterns in the UV2 and VIS ranges. Considering the complexity of the temporal and spatial changes, in the summary Table 6 we provide only indicative estimates of the RA-affected rows. For example, currently about $33 \%$ of the UV2 rows are affected in the southern-equatorial parts of the OMI orbit. This increases to $\sim 57 \%$ in the northern hemisphere. These estimates are comparable to the VIS numbers, though very different in the UV1 case, where all rows are affected at the middle-to-high northern latitudes. Users of the OMI data are advised to discard the affected rows in accordance with the warning flags provided in the L1B products, though one may notice a broad range of RA sensitivity among the different L2 OMI products. 
As appears from the presented data, OMI ages very gracefully, showing remarkably low radiance degradation and high wavelength stability. The most serious concern is the developing row anomaly. However, its spread has stabilized since the last rapid development in the fall of 2011. Assuming status quo, one may expect for the instrument to deliver useful science data for 5-10 additional years.

Data availability. All analysis results presented in this article are based on the OMI Level 1B data. This dataset is publicly available at NASA's Goddard Earth Sciences Data and Information Services Center (GES-DISC, 2004). 


\section{Appendix A: Wavelength registration approach}

This appendix describes how the wavelength registration of OMI was performed during the on-ground calibration period and how it is executed in flight. During the on-ground calibration period the wavelength calibration was performed using a PtCrNeAr spectral line source. In the analysis of the calibration data, small wavelength windows were used that contained exactly one spectral line, whose wavelength is accurately known. Five windows were used In the UV1 channel, and nine windows in the UV2 and VIS channels. In such a window the spectral calibration was performed by fitting the measured spectrum to a Gaussian function in the UV1 channel. In the UV2 and VIS channels a sum of a Gaussian and a function with a flatter top was used:

$F(x)=A_{0} e^{-\left(\frac{x-x_{0}}{W_{0}}\right)^{2}}+A_{1} e^{-\left(\frac{x-x_{1}}{W_{1}}\right)^{4}}$.

The fits were performed in pixel space. In this way for all windows the peak position of the spectral line in pixel space was determined. The peak positions together with their wavelengths are fitted to a fourth-order polynomial to obtain the wavelength scale. This wavelength scale is used to calculate the wavelength of a given column number in the L1B processor.

During the mission the wavelength assignment is done using two methods. The wavelength coefficients are obtained for both the radiance and irradiance measurements. In the first method the wavelength assignment is based on prelaunch and early in-orbit wavelength calibration parameters. These parameters are modified as a function of optical bench temperature:

$$
\begin{aligned}
c_{2, n}(j) & =c_{n}(j)+d_{n}(j)\left(T_{\mathrm{OPB}}-T_{\mathrm{sc} \_ \text {ref }}\right) \\
& +e_{n}(j)\left(T_{\mathrm{OPB}}-T_{\mathrm{sc} \_ \text {ref }}\right)^{2},
\end{aligned}
$$

where $c_{n}(j)$ are the pre-launch wavelength calibration parameters, $T_{\mathrm{OPB}}$ is the temperature of the optical bench during the measurement, $T_{\mathrm{sc} \_ \text {ref }}$ is a reference temperature, $d_{n}(j)$ and $e_{n}(j)$ are wavelength temperature coefficients, and $j$ is row number. In the UV2 and VIS channel this function is corrected for wavelength shifts that result from inhomogeneous slit illumination. This correction makes use of small pixel data. Normal radiance and irradiance data are data that have been binned on the CCD and co-added in the electronics unit. Both operations result in increased signal-to-noise ratio at the expense of spatial and temporal resolution. It also helps in reducing the data rate to ground. A typical binning factor is 8 , and a typical co-adding factor is 5 . Small pixel data is data that has not been co-added. The correction function for inhomogeneous slit illumination is as follows:
$Q(j)=2 \cdot \frac{S_{\mathrm{SPC}}\left(N_{\text {co-addition }}-1, j\right)-S_{\mathrm{SPC}}(0, j)}{S_{\mathrm{SPC}}\left(N_{\text {co-addition }}-1, j\right)+S_{\mathrm{SPC}}(0, j)}$,

$c_{2, n}(j)=c_{2, n}(j)+b_{\mathrm{OPF}}(j) Q(j)$,

where $S_{\mathrm{SPC}}$ is a matrix containing small pixel data, $N_{\text {co-addition }}$ is the number of co-additions used for this measurement, $b_{\mathrm{OPF}}(j)$ are the transformation parameters and $\mathrm{j}$ is row number. With the wavelength coefficients $c_{2, n}(j)$ a wavelength map is calculated using

$\lambda(i, j)=\sum_{n=0}^{N} c_{2, n}(j)\left(i-i i_{\mathrm{ref}}\right)^{n}$,

where $i$ is column number, $j$ is row number, $N$ is number of polynomial coefficients (typically 4 ) and $i i_{\text {ref }}$ is a reference column for the spectral calibration polynomial coefficients. The wavelength coefficients $c_{2, n}(j)$ and the reference column are stored in the L1B radiance and irradiance output products.

For the second method, the wavelength calibration is performed by fitting an accurately known solar spectrum, an ozone absorption spectrum and a Ring spectrum to the measured spectrum. The reference spectrum is divided in 8 windows for the UV1 channel, 18 windows for the UV2 channel and 22 windows for the VIS channel. These windows contain characteristic features of the solar spectrum like Fraunhofer lines. For each window a fit of the measured spectrum with the reference spectrum is performed in wavelength space. The boundaries of a window are given by $\lambda_{\min }$ and $\lambda_{\max }$. An initial estimate of the wavelength scale is given by $\lambda(i, j)$, where $i$ and $j$ are the column and row index of the CCD detector. The result of the fit process will be a wavelength scale $\lambda$, where

$\lambda^{\prime}(\lambda(i, j))=\lambda(i, j)+\lambda_{\text {shift }}+\left(\lambda(i, j)-\lambda_{\text {center }}\right) \lambda_{\text {squeeze }}$,

$\lambda_{\text {center }}=\frac{\lambda_{\min }+\lambda_{\max }}{2}$,

and $\lambda_{\text {shift }}$ and $\lambda_{\text {squeeze }}$ are non-linear fit parameters. The fit process is executed by minimizing the spectral calibration merit function

$\chi_{\text {window }}^{2}=\sum_{\lambda=\lambda_{\min }}^{\lambda_{\max }}\left(\frac{S_{\text {measurement }}(\lambda)-S_{\text {fit }}\left(\lambda^{\prime}\right)}{\sigma(\lambda)}\right)^{2}$,

where

$S_{\text {measurement }}(\lambda)={ }^{e} \log S(\lambda(i, j))$, 
$S(\lambda(i, j))=S(i, j)$

which is the signal at detector location $(i, j)$, and

$\sigma(\lambda)=\frac{\operatorname{Noise}(\lambda(i, j))}{S(\lambda(i, j))}$.

The fit function is

$$
\begin{aligned}
S_{\text {fit }}\left(\lambda^{\prime}\right) & =a_{0} \mathrm{sf}_{\text {DOAS }, 0}^{e} \log \left(I_{\text {sun }}\left(\lambda^{\prime}\right)\right)-a_{1} \operatorname{sf}_{\text {DOAS }, 1} \sigma_{\text {ozone }}\left(\lambda^{\prime}\right) \\
& -a_{2} \mathrm{sf}_{\text {DOAS }, 2} \operatorname{Ring}_{\text {DOAS }}\left(\lambda^{\prime}\right) \\
& +\sum_{n=3}^{5} a_{n}\left(\lambda^{\prime}-\lambda_{\text {center }}\right)^{n-3} \operatorname{sf}_{\text {DOAS }, n},
\end{aligned}
$$

where $I_{\text {sun }}\left(\lambda^{\prime}\right)$ is a high-resolution Sun spectrum, $\sigma_{\text {ozone }}\left(\lambda^{\prime}\right)$ is an ozone absorption spectrum, $\operatorname{Ring}_{\mathrm{DOAS}}\left(\lambda^{\prime}\right)$ is a $\operatorname{Ring}$ spectrum, $\mathrm{a}_{n}$ are the fit parameters and $\mathrm{sf}_{\mathrm{DOAS}, n}$ are the scaling parameters. The Sun spectrum, ozone absorption spectrum and Ring spectrum need to be pre-convolved with the OMI instrument spectral response function. The scaling parameters $\mathrm{sf}_{\mathrm{DOAS}, n}$ are used to keep the fit parameters $a_{n}$ in a range in which the derivative calculation during the fit process provides optimal performance. After minimization of the merit function, a column-wavelength-precision triplet is calculated for each window $w$ :

$i_{\mathrm{win}}(w)=\frac{i_{\min }(w)+i_{\max }(w)}{2}$,

where $i_{\min }(w)$ and $i_{\max }(w)$ are the columns with a wavelength closest to $\lambda_{\min }$ and $\lambda_{\max }$ of the window,

$\lambda_{\text {win }}(w)=\lambda^{\prime}\left(\lambda\left(i_{\operatorname{win}}(w), j\right)\right)$.
The precision $\sigma_{\text {win }}(w)$ is taken equal to the covariance of the result of the minimization of the merit function. The sets of triplets of all windows in a row are taken for a second polynomial fit. The polynomial is fitted by minimization of the following merit function:

$\chi_{\text {row }}^{2}=\sum_{w=0}^{W}\left(\frac{\lambda_{\text {win }}(w)-\lambda_{\text {fit }}\left(i_{\text {win }}(w)\right)}{\sigma_{\text {win }}(w)}\right)^{2}$,

where

$\lambda_{\mathrm{fit}}(i)=\sum_{p=0}^{P} c_{\mathrm{fit}, p}(j)\left(i-i i_{\mathrm{ref}}\right)^{p}$

and $c_{\mathrm{fit}, p}(j)$ are the polynomial coefficients for a row $\mathrm{j}$, $\mathrm{ii}_{\mathrm{ref}}$ is a reference column for the spectral calibration polynomial coefficients, $P$ is the number of polynomial coefficients and $W$ is the number of windows. With these coefficients the wavelength of a pixel can be calculated for a given column row index $(i, j)$. Typically the polynomial is fourth order. For every row a set of polynomial coefficients is calculated. The fitting procedure is executed both for radiance and irradiance measurements. For irradiance measurements the ozone and Ring absorption spectra are excluded from the fit function. The wavelength coefficients $c_{\text {fit }, p}(j)$ and the reference column are stored in the L1B calibration output product. 
Competing interests. The authors declare that they have no conflict of interest.

Acknowledgements. The authors would like to acknowledge the work of the large team of colleagues at KNMI, FMI, NASA and in industry that have contributed to the success of the OMI instrument. This work could not have been done without funding from the Netherlands Space Organization and NASA.

Edited by: V. Sofieva

Reviewed by: M. Dobber and one anonymous referee

\section{References}

Ahmad, Z., Bhartia, P. K., and Krotkov, N.: Spectral properties of backscattered UV radiation in cloudy atmospheres, J. Geophys. Res., 109, D01201, doi:10.1029/2003JD003395, 2004.

Bazalgette Courrèges-Lacoste, G., Arcioni, M., Meijer, Y., Bézy, J.L., Bensi, P., and Langen, J.: Sentinel-4: The Geostationary Component of the GMES Atmospheric Monitoring Mission, Proceedings of the 7th ICSO (International Conference on Space Optics) 2008, Toulouse, France, 14-17 Oct., 2008.

Bertaux, J. L., Kyrölä, E., Fussen, D., Hauchecorne, A., Dalaudier, F., Sofieva, V., Tamminen, J., Vanhellemont, F., Fanton d'Andon, O., Barrot, G., Mangin, A., Blanot, L., Lebrun, J. C., Pérot, K., Fehr, T., Saavedra, L., Leppelmeier, G. W., and Fraisse, R.: Global ozone monitoring by occultation of stars: an overview of GOMOS measurements on ENVISAT, Atmos. Chem. Phys., 10, 12091-12148, doi:10.5194/acp-10-12091-2010, 2010.

Bovensmann, H., Burrows, J. P., Buchwitz, M., Frerick, J., Noel, S., Rozanov, V. V., Chance, K. V., and Goede, A. P. H.: SCIAMACHY-Mission objectives and measurement modes, J. Atmos. Sci., 56, 127-150, 1999.

Burrows, J. P., Weber, M., Buchwitz, M., Rozanov, V., LadstätterWeißenmayer, A., Richter, A., Debeek, R., Googen, R., Bramstedt, K., Eichman, K.-U., Eisinger, M., and Perner, D.: The Global Ozone Monitoring Experiment (GOME): Mission concept and first scientific results, J. Atmos Sci., 56, 151-175, 1999.

Cebula, R. P., Park, H., and Heath, D. F.: Characterization of the Nimbus-7 SBUV Radiometer for the Long-Term Monitoring of Stratospheric Ozone, J. Atmos. Ocean. Technol., 5, 215-227, 1988.

Chance, K., Liu, X., Suleiman, R. M., Flittner, D. E., Al-Saadi, J., and Janz, S. J.: Tropospheric emissions: Monitoring of pollution (TEMPO), Proceedings of SPIE, Vol. 8866, Earth Observing Systems XVIII, 88660D (September 23, 2013), San Diego, CA, USA, 2013.

Deland, M. and Marchenko, S.: The solar chromospheric Ca and $\mathrm{Mg}$ indices from Aura OMI, J. Geophys. Res.-Atmos., 118, 3415-3423, 2013.

Dobber, M. R., Dirksen, R., Levelt, P., Oord, G. H. J. van den, Voors, R., Kleipool, Q., Jaross. G., Kowalewski, M., Hilsenrath, E., Leppelmeier, G., de Vries, J., Dierssen, W., and Rozemeijer, N.: Ozone Monitoring Instrument Calibration, IEEE T. Geosci. Remote., 44, 1209-1238, 2006.

Dobber, M. R., Kleipool, Q., Dirksen, R., Levelt, P. F., Jaross, G., Taylor, S., Kelly, T., Flynn, L., Leppelmeier, G., and
Rozemeijer, N.: Validation of ozone monitoring instrument level 1b data products, J. Geophys. Res., 113, D15S06, doi:10.1029/2007JD008665, 2008a.

Dobber, M., Voors, R., Dirksen, R., Kleipool, Q., and Levelt, P.: The High-Resolution Solar Reference Spectrum between 250 and $550 \mathrm{~nm}$ and its Application to Measurements with the Ozone Monitoring Instrument, Sol. Phys., 249, 281-291, 2008 b.

GES-DISC: Goddard Earth Sciences Data and Information Services Center, available at: https://disc.gsfc.nasa.gov/uui/ datasets? processingLevel=1\&keywords=AuraOMI (last access: 8 May 2017), 2004.

Haffner, D.: OMTO3 Release Specific Information, available at: http://disc.sci.gsfc.nasa.gov/Aura/data-holdings/OMI/ documents/v003/OMTO3_ReleaseDetails_V003.doc (last access: 4 July 2016), 2012.

Jaross, G., Cebula, R. P., DeLand, M. T. , and Steinfeld, K.: Backscatter Ultraviolet Instrument Solar Diffuser Degradation, Proc. SPIE, 3427, 432, 1998.

Kim, J., Kim, M., Jeong, U., Choi, M., and Go, S.: GK-2B Atmospheric Environment Mission, GEMS : Atmospheric Composition Monitoring in High Temporal and Spatial Resolution, The 1st KMA International Meteorological Satellite Conference, Seoul, Korea, 16-18 November, 2015.

Kurosu, T. P., Chance, K., and Sioris, C. E.: Preliminary results for $\mathrm{HCHO}$ and $\mathrm{BrO}$ from the EOS-Aura Ozone Monitoring Instrument, Passive Optical Remote Sensing of the Atmosphere and Clouds IV, 116, 116-123, 2004.

Kyrölä, E., Tamminen, J., Leppelmeier, G. W., Sofieva, V., Hassinen, S., Bertaux, J. L., Hauchecorne, A., Dalaudier, F., Cot, C., Korablev, O., Fanton d'Andon, O., Barrot, G., Mangin, A., Théodore, B., Guirlet, M., Etanchaud. F., Snoeij, P., Koopman, R., Saavedra, L., Fraisse, R., Fussen, D., and Vanhellemont, F.: GOMOS on Envisat: An overview, Adv. Space Res., 33, 1020 1028, 2004.

Levelt, P. F., Oord, G. H. J. van den, Dobber, M. R., Mälkki, A., Visser, H., Vries, J. de, Stammes, P., Lundell, J., and Saari, H.: The Ozone Monitoring Instrument, IEEE T. Geosci. Remote, 44, 1093-1101, 2006.

Marchenko, S. and Deland, M.: Solar spectral irradiance changes during cycle 24, Astrophys. J., 789, 117-134, 2014.

Marchenko, S., Krotkov, N. A., Lamsal, L. N., Celarier, E. A., Swartz, W. H., and Bucsela, E. J.: Revising the slant column density retrieval of nitrogen dioxide observed by the Ozone Monitoring Instrument, J. Geophys. Res.-Atmos., 120, 5670-5692, 2015.

McPeters, R. D., Bhartia, P. K., Krueger, A. J., Herman, J. R., Wellemeyer, C. G., Seftor, C. J., Jaross, G., Torres, O., Moy, L., Labow, G., Byerly, W., Taylor, S. L., Swissler, T., and Cebula, R. P.: Earth Probe Total Ozone Mapping Spectrometer ('TOMS) Data Product's User's Guide, NASA Technical Publication 1998-206895, 1998.

Oord, G. H. J. van den, Rozemeijer, N. C., Schenkelaars, V., Levelt, P. F., Dobber, M. R., Voors, R. H. M., Claas, J., de Vries, J., ter Linden, M., de Haan, C., and van den Berg, T.: OMI level 0 to $1 \mathrm{~b}$ processing and operational aspects, IEEE T. Geosci. Remote, 44, 1380-1397, 2006.

Seftor, C. J., Jaross, G., Kowitt, M., Haken, M., Li, J. ,and Flynn, L. W.: Post-launch performance of the Suomi NPP Ozone Mapping and Profiler Suite (OMPS) nadir sensors, J. Geophys. Res.Atmos., 119, 4413-4428, 2013. 
Sierk, B. Bézy, J.-L., Caron, J., Meynard, R., Veihelmann, B., Ingmann, P.: The GMES Sentinel-5 mission for operational atmospheric monitoring: Status and Developments, Proceedings of the ICSO (International Conference on Space Optics), Ajaccio, Corse, France, 9-12 Oct., 2012.

Snow, M., McClintock, W. E., Woods, T. N., White, O. R., Harder, J. W., and Rottman, G.: The Mg II index from SORCE, Sol. Phys., 230, 325-344, 2005

TMCF: available at: http://projects.knmi.nl/omi/research/ (last access: 8 May 2017), 2006.

van Geffen, J. H. G. M., Boersma, K. F., Van Roozendael, M., Hendrick, F., Mahieu, E., De Smedt, I., Sneep, M., and Veefkind, J. P.: Improved spectral fitting of nitrogen dioxide from OMI in the 405-465 nm window, Atmos. Meas. Tech., 8, 1685-1699, doi:10.5194/amt-8-1685-2015, 2015.
Vasilkov, A., Joiner, J., Spurr, R., Bhartia, P. K., Levelt, P., and Stephens, G.: Evaluation of the OMI cloud pressures derived from rotational Raman scattering by comparisons with other satellite data and radiative transfer simulations, J. Geophys. Res., 113, D15S19, doi:10.1029/2007JD008689, 2008.

Veefkind, J. P., Aben, I., and Levelt, P. F.: TROPOMI on Sentinel$5 \mathrm{P}$ the next generation atmospheric composition spectrometer, Proceedings of ATMOS 2012, Advances in Atmospheric Science and Applications, Bruges, Belgium, June 18-22, 2012. 